\title{
4. THEORETICAL DESCRIPTION OF THE INTERSTELLAR MEDIUM
}

\author{
Introductory Report
}

(Tuesday, September 9, 1969)

GEORGE B. FIELD*

Dept. of Applied Mathematics and Theoretical Physics, Cambridge University, Cambridge, U.K.

\section{Introduction}

As excellent reviews have appeared recently (Spitzer, 1968a; Pikel'ner, 1968a) we shall consider here only some special topics where progress is being made - thermal properties of $\mathrm{HI}$ and $\mathrm{HII}$ clouds, and shock waves. The components of the medium which are dynamically important are neutral atoms, charged ions and electrons, grains, cosmic rays, magnetic field, and light. In Table I we indicate the degree of

\section{TABLE I}

Momentum coupling between components

( $\mathrm{S}=$ Strong, $\mathbf{M}=$ Medium, $\mathrm{W}=$ Weak)

\begin{tabular}{lllllll}
\hline Component & Atoms & Ions & Grains & Cosmic rays & Light & Magnetic field \\
Atoms & S & S & M & W & W & W \\
Ions & S & S & M & W & W & S \\
Grains & M & M & W & W & M & M \\
Cosmic rays & W & W & W & W & W & S \\
Light & W & W & M & W & W & W \\
Magnetic field & W & S & M & S & W & - \\
\hline
\end{tabular}

momentum coupling among these components. The main mass of the medium, in ions and atoms, is well coupled through collisions, with a mean free path $\lambda \approx 10^{-3} \mathrm{pc}$, and may usually be treated hydrodynamically. Parker (1965) has shown that for many purposes the cosmic-rays can be considered as a separate gas, strongly coupled to the magnetic field. The coupling of the atom-ion gas to the magnetic field is through the magnetic forces on the ions, which transmit the forces to the neutral atoms by collisions unless the differential stresses on the two components are unusually large. Grains are not coupled strongly to any other component, as their stopping distances are of the order of parsecs and their gyro-periods of the order of $10^{4} \mathrm{yr}$. The coupling of grains to radiation pressure can lead to interesting differential effects. Light is important in carrying away thermal energy but does not critically affect the momentum balance of the gas. A possible exception is the Ly- $\alpha$ radiation field discovered by Kurt and Sunyaev (1967) which has a pressure of $10^{-13}$ dyne $\mathrm{cm}^{-2}$. If this phenomenon is common in interstellar space, it could have effects.

* Usual address: Dept. of Astronomy, University of California, Berkeley, Calif., U.S.A. 
Most work has been on the large-scale motion of the atom-ion gas, includes magnetic stresses, and assumes that the magnetic field is frozen in. Such studies include equilibrium configuration in the galactic magnetic field, cloud collisions, expansions of $\mathrm{HII}$ regions and supernova shells, and perturbation calculations aimed at discovering instabilities (gravitational, thermal, hydromagnetic). Many of these calculations have paid insufficient attention to the effects of cosmic-ray pressure. Similarly, insufficient work has been done on the dynamical effects of radiation pressure on grains.

\section{Thermal Properties of HI Regions and Formation of Clouds}

Spitzer calculated thermal equilibrium conditions in the 1940's. He concluded that the main heating is due to photo-electrons ejected from carbon atoms, and that the main cooling is by infrared emission following collisional excitation of the fine-structure levels of various ions. Spitzer's equilibrium temperatures $(\approx 20 \mathrm{~K})$ are too low to explain the $21-\mathrm{cm}$ result that $\left\langle T^{-1}\right\rangle^{-1} \approx 100 \mathrm{~K}$, therefore Kahn (1955) suggested that the temperature may be maintained by shocks associated with supersonic cloud collisions. We shall see below that this mechanism appears inadequate to maintain the temperature. Hayakawa et al. (1961) showed that if low-energy (1 to $100 \mathrm{MeV})$ cosmic rays are present in sufficient numbers, the heating by the secondary electrons they produce would explain the temperatures of clouds. Field (1962) showed that the observed cosmic rays with $E>1 \mathrm{GeV}$ would heat any low-density gas to $10^{4} \mathrm{~K}$ or above, so that there could be pressure equilibrium between two phases: a rarefied, hot intercloud medium and dense, cool clouds. This explanation of the stability of clouds had been suggested by Spitzer (1951). Pikel'ner (1967) revived the discussion, showing that the heating by cosmic rays and the partial ionization of otherwise neutral $\mathrm{HI}$ explains various observations, including the existence of hot $\mathrm{HI}(21-\mathrm{cm})$ regions, Faraday rotation, and long-wavelength radio absorption. Pikel'ner included the heating due to Coulomb collisions of cosmic rays with ambient thermal electrons and discussed extensively the possibility and the consequences of the existence of three thermal phases in the interstellar gas: a stable, hot phase of low density $\left(n \lesssim 10^{-1} \mathrm{~cm}^{-3}\right.$, $T \gtrsim 6000 \mathrm{~K})$, a stable, cool phase of high density $\left(n \gtrsim 1 \mathrm{~cm}^{-3}, T \lesssim 300 \mathrm{~K}\right)$, and an intermediate, thermally unstable phase in which $\mathrm{d} p / \mathrm{d} \varrho<0$.

Spitzer and Tomasko (1968) investigated the efficiency of cosmic-ray heating by calculating the energy put into the secondary electrons and subtracting the energy lost by the secondaries through excitation of optical transitions. Later, Spitzer and Scott (1969) (to be called SS) extended these calculations to include elastic collisions between the secondary electrons and the ambient thermal electrons, and Coulomb collisions between cosmic rays and ambient electrons.

Field et al. (1969) (FGH) and Goldsmith et al. (1969) using a different method, also included the efficiency of the secondaries in their calculations and calculated equilibrium temperatures (heating =cooling) using cooling processes and crosssections somewhat different from those of Pikel'ner and of Spitzer and Tomasko. 
They found results qualitatively similar to Pikel'ner's and showed that Pikel'ner's model also explains the electron density observed by pulsar dispersion measurements. They concluded that if agreement with cloud temperatures is to be obtained, depletion of cooling elements on grains is to be postulated. The latter effect might then explain the low abundance of sodium and calcium as deduced from interstellar absorptionline observations. Hjellming et al., (1969) (HGG), using somewhat different cooling processes, applied the efficiency calculations of Spitzer and Scott and obtained thermal equilibria in agreement with FGH. Silk and Werner (1969) have suggested that ionization by $\mathrm{X}$-rays $<1 \mathrm{keV}$ could supplement or replace low-energy cosmic rays as a heating and ionizing agent.

Figure 1 shows the amount of energy delivered to the gas and the total number of electrons produced per primary ionization as a function of $n_{e} / n$. The values of SS are the most reliable. Figure 2 shows the equilibrium temperatures derived. The results have all been normalized to a particular value of the primary ionization rate $\zeta$ of hydrogen found by FGH to be in good agreement with observation. (FGH showed that all processes are proportional to this function of the cosmic-ray spectrum, so that the detailed form of the latter, which is unknown, is irrelevant). Three of the calculations agree fairly well in view of the somewhat different assumptions about $\zeta_{\text {eff }}$ and

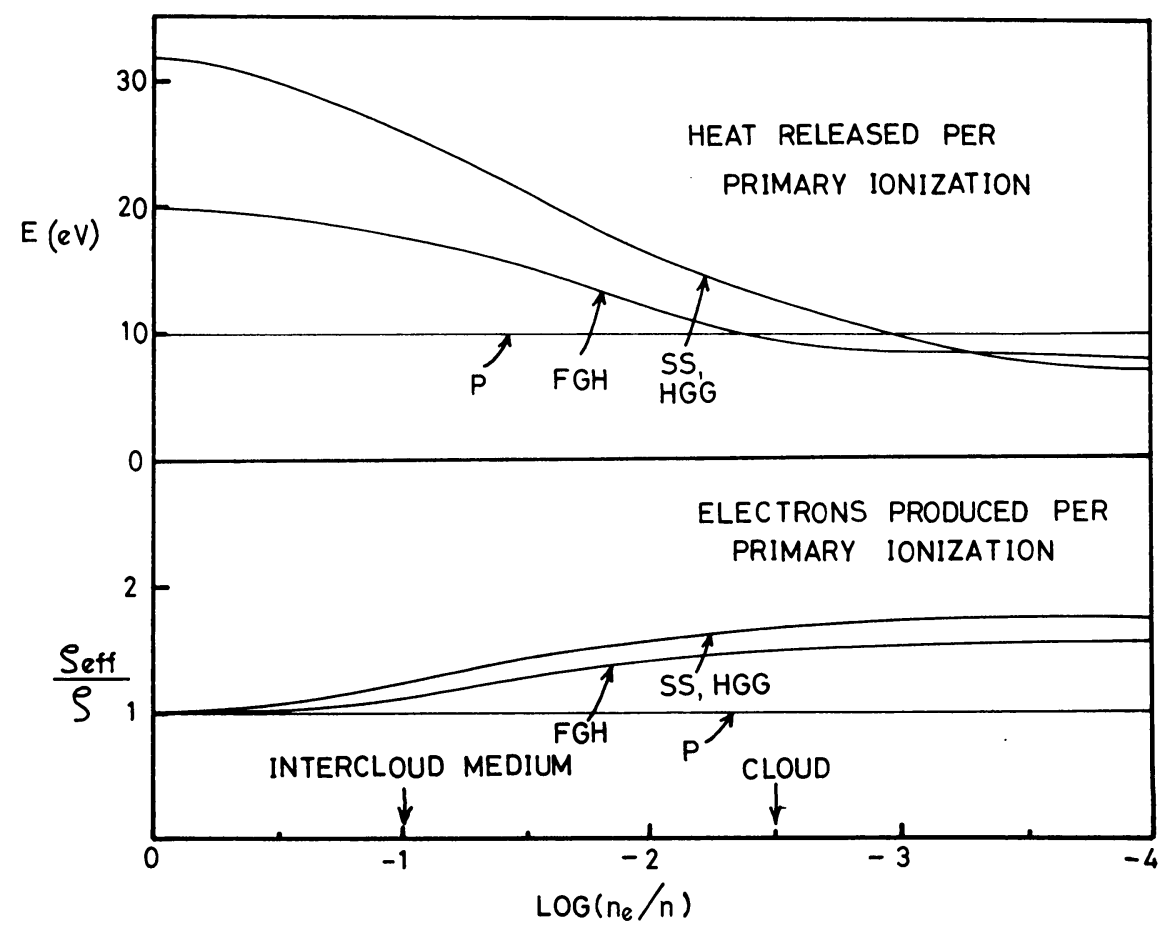

Fig 1. Heat released and number of electrons produced per primary ionization of a neutral hydrogen atom. $\mathrm{P}=$ Pikel'ner (1967), FGH = Field, Goldsmith, and Habing (1969), SS = Spitzer and Scott (1969), HGG $=$ Hjellming, Gordon, and Gordon (1969). $\zeta$ is the rate of primary ionization per neutral hydrogen atom; $\zeta$ eff includes in addition ionization by energetic secondary electrons. 


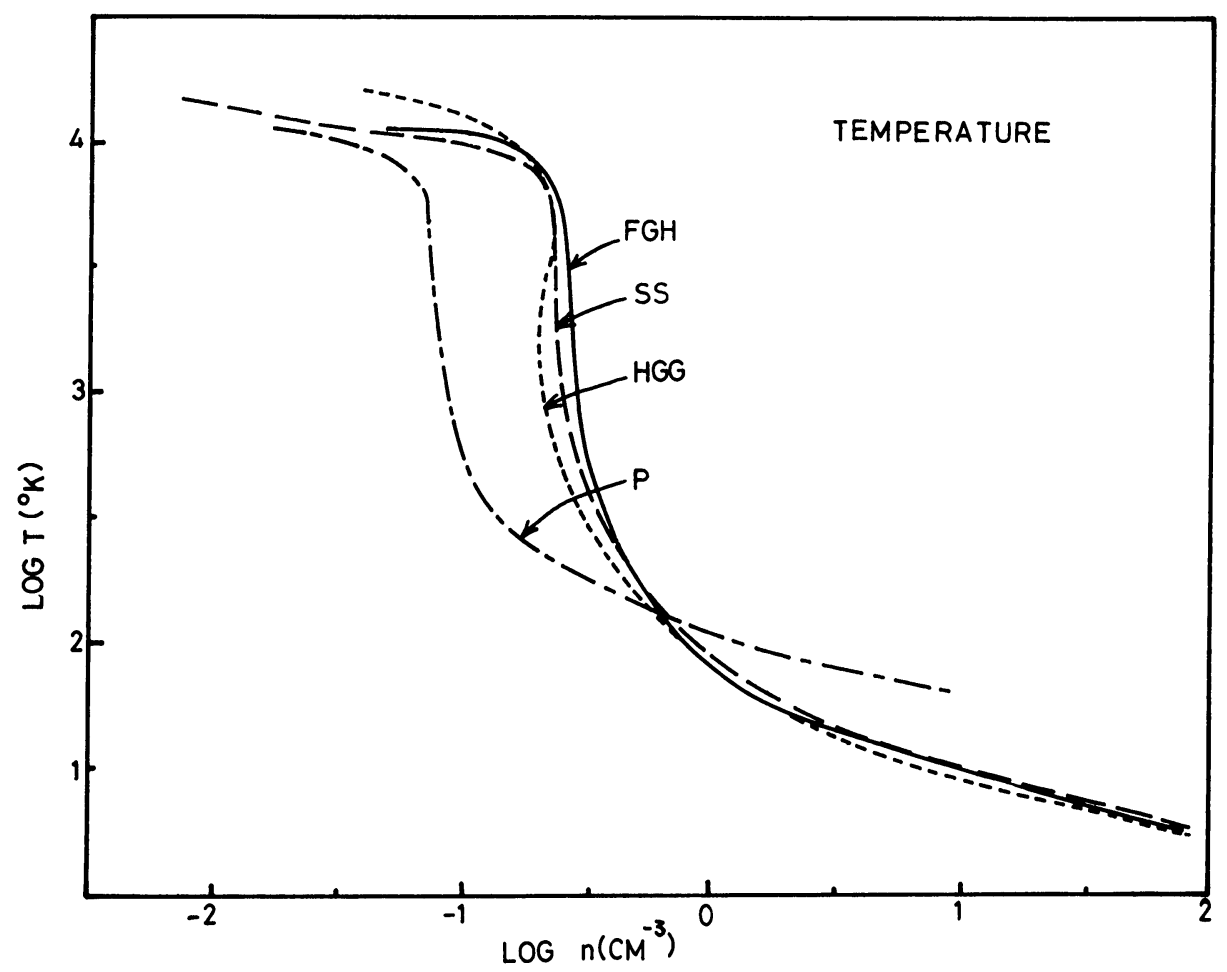

Fig. 2. Temperature for which the cosmic ray heating equals the cooling by inelastic, thermal collisions as a function of density $n$. Results of various authors are normalized to one value of $\zeta\left(=4 \times 10^{-16} \mathrm{sec}^{-1}\right)$. See Figure 1 for explanation on the names of the authors.

$\Delta E$ (Figure 1), and about the cooling mechanisms. The results of Pikel'ner agree qualitatively, and the quantitative differences may probably be explained by the fact that certain of his cross sections were only estimated.

The curves can be understood qualitatively as follow. As $n$ drops, the cooling $\left(\propto n^{2}\right)$ decreases faster than the heating $(\propto n)$ and $T$, which influences only the cooling, must rise to compensate for the difference. In the range $10^{3}$ to $5 \times 10^{3} \mathrm{~K}$, fine-structure cooling is relatively insensitive to $T$, and $T$ must rise rapidly to about $10^{4} \mathrm{~K}$, where Ly- $\alpha$ cooling becomes important. FGH included the ${ }^{3} P_{-}{ }^{1} D$ transition of $\mathrm{O} I$ at $1.96 \mathrm{eV}$ $(E / k=22800 \mathrm{~K})$, while SS and HGG included the ${ }^{3} P_{-}{ }^{1} D$ transition of $\mathrm{N}$ II at $1.90 \mathrm{eV}$ $(E / k=22100 \mathrm{~K})$ as well. The conclusion that N II should not contribute significantly (FGH) is strengthened by the agreement of HGG, SS, and FGH. All authors omitted what could possibly be an important mechanism for cooling the intercloud medium, i.e., electron excitation of a ${ }^{4} F_{9 / 2}$-level of $\mathrm{Fe}^{+}$with $E / k=2700 \mathrm{~K}$. Pottasch (1968) estimates $\Omega=15$ for this transition, and if $\mathrm{Fe} / \mathrm{O}=0.1$ (Pottasch, 1963; Swings, 1965; Gary et al., 1969), rather than the 0.01 assumed by FGH, we find that this transition should be quite important in the range of several thousand degrees and would thus depress the temperature of the intercloud medium (see below). 


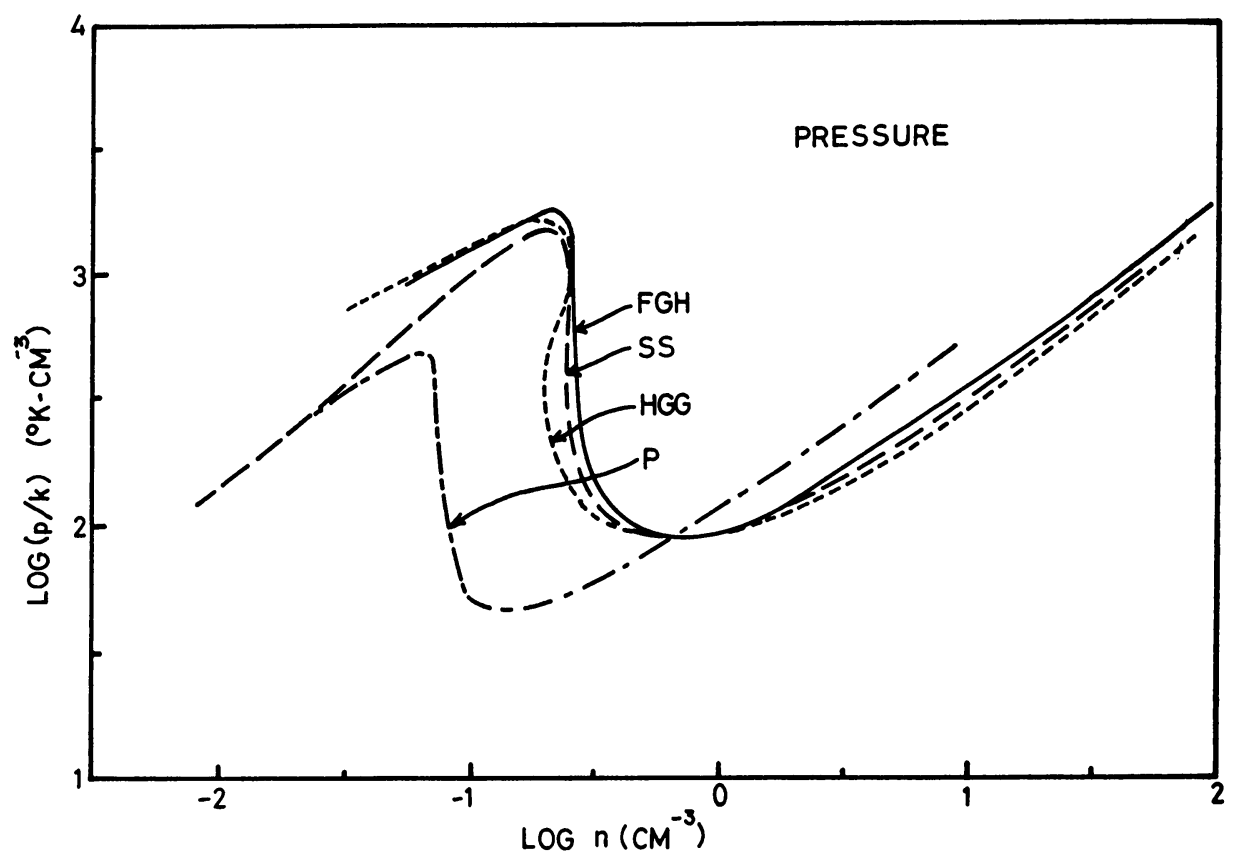

Fig. 3. The pressure of the interstellar medium as a function of total nuclear density. See Figures 1 and 2 for explanation.

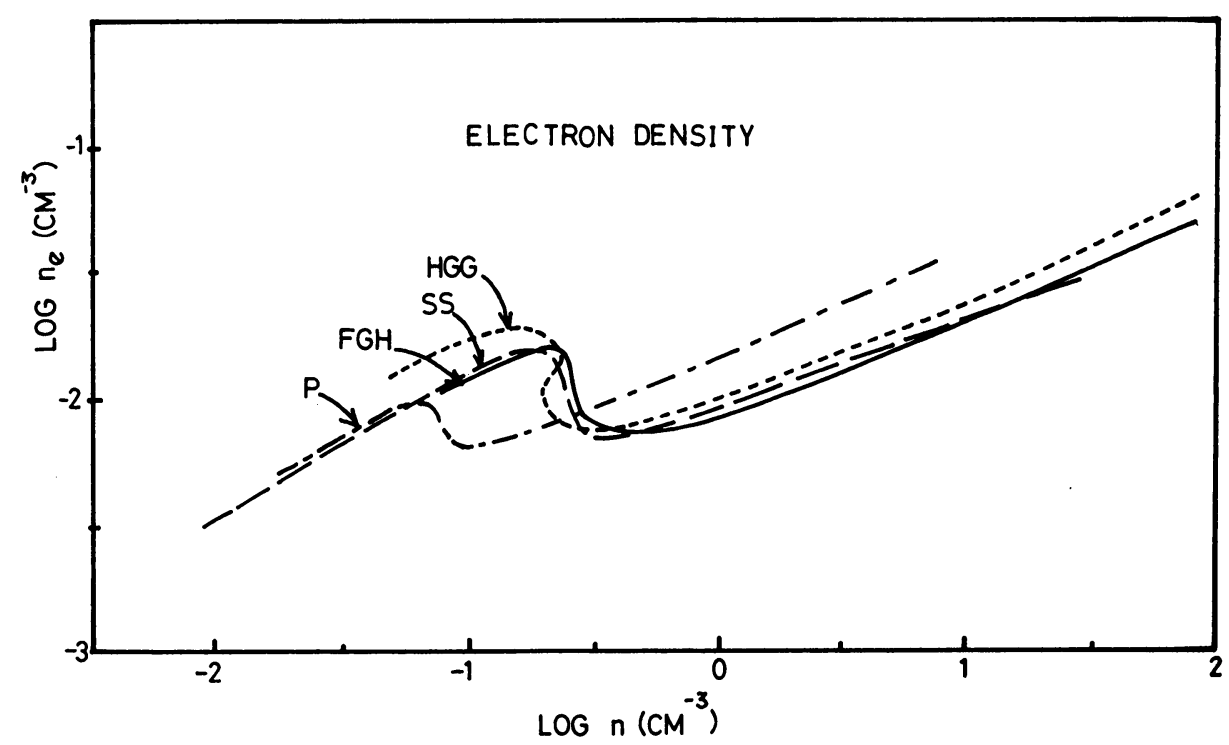

Fig. 4. Electron density in the interstellar medium as a function of total nuclear density. See Figures 1 and 2 for explanation. 
Figure 3 shows the gas pressure $p / k=\left(n+n_{e}\right) T$; and Figure 4, the electron density $n_{e}$. The latter is about $10^{-2} \mathrm{~cm}^{-3}$, in agreement with observations of pulsar dispersion and low-frequency radio absorption. In every case there is a pressure maximum; if gas in the high-temperature phase at the left of the maximum were to be slowly compressed, no equilibrium could be found beyond the critical point whose parameters $\left(p_{c}, n_{c}, T_{c}\right)$ are listed in Table II. FGH interpret this to mean that in our local neigh-

TABLE II

Parameters of critical point $\left(\zeta=4 \times 10^{-16} \mathrm{sec}^{-1}\right)$

\begin{tabular}{lllcl} 
Author & $n_{c}\left(\mathrm{~cm}^{-3}\right)$ & $T_{c}(\mathrm{~K})$ & $p_{c}\left(\mathrm{~cm}^{-3} \mathrm{~K}\right)$ & $n_{e c}\left(\mathrm{~cm}^{-3}\right)$ \\
\hline P & & & & \\
FGH & 0.062 & 7000 & 430 & 0.009 \\
SS & 0.21 & 7800 & 1800 & 0.016 \\
HGG & 0.21 & 7000 & 1500 & 0.015 \\
& 0.19 & 9000 & 1700 & 0.016
\end{tabular}

References: $P=$ Pikel'ner (1967); FGH $=$ Field, Goldsmith, and Habing (1969); SS $=$ Spitzer and Scott (1969); HGG $=$ Hjellming, Gordon, and Gordon (1969).

borhood gas must occur in the low-temperature phase. This gas forms the cool clouds that produce at $21 \mathrm{~cm}$ all of the absorption lines and part of the emission lines. The argument is as follows. Consider a particular tube of force, which on the Parker (1968a) model of the magnetic field (see below) loops out of the plane. Along the tube we must have hydrostatic equilibrium in the galactic gravitational field, $K_{z}$. At large $z$, the density is low and the gas is in the hot stable phase. If one can integrate the hydrostatic equilibrium equation to the lowest point on the flux tube without exceeding $p_{c}$, the entire tube could be filled with hot gas, but if the weight of the gas is too great, one will reach $p_{c}$ first. Since $\mathrm{d} p / \mathrm{d} z<0$ in hydrostatic equilibrium, a phase transition to the stable cool phase must occur and the gas will form normal interstellar clouds. FGH showed that the amount of gas observed in our local neighborhood is too great to be accommodated in the hot stable phase, so that clouds must occur. Pikel'ner (1967) also took this point of view, and pointed out that if the total density between arms is much lower, there may be no clouds there.

This model predicts the existence of a rarefied, hot $\mathrm{HI}_{\mathrm{I}}$ intercloud medium. Recent observations of the $21-\mathrm{cm}$ line in absorption confirm this prediction and give $T>$ 1000K (Clark 1965; Radhakrishnan and Murray, 1969). Observations of the same line in emission also indicate the existence of hot H I gas (Heiles, 1967a; Habing, 1969; Grahl et al., 1969). However, according to Heiles (1967a) the velocity dispersion of the line profile indicates $T \leqslant 4000 \mathrm{~K}$, which is much less than that predicted by theory for the stable hot phase $(T>8000 \mathrm{~K}$, see Table II). Mechanisms, such as iron cooling, which might reduce $T_{c}$ (the temperature at the critical point), are therefore of particular interest. HGG suggest that a way out of this problem is to take the intercloud 
medium to be the intermediate unstable phase. We have two objections to this procedure. Away from the plane, where $K_{z}$ is significant, hydrostatic equilibrium demands that $\mathrm{d} p / \mathrm{d} z<0$, and since $(\mathrm{dp} / \mathrm{d} \varrho)<0$ in the intermediate phase, a region containing this phase would have $(\mathrm{d} \varrho / \mathrm{d} z)>0$, which is Rayleigh-Taylor unstable (Layzer, $1967)$ on a time scale $\left(\mathrm{d} K_{z} / \mathrm{d} z\right)^{-1 / 2}=1.3 \times 10^{7} \mathrm{yr}$. Even if $z$ is small, and $K_{z} \propto z$, gas in the intermediate phase is thermally unstable (Field, 1965). According to Pikel'ner (1967), intermediate-phase gas may be present while the long wavelengths required to make interstellar clouds are developing, since the time scale for development of long wavelengths is about $\lambda / c \gg$ thermal time scale ( $c$ is the sound speed). However, this overlooks the fact that shorter wavelengths will develop faster, namely on the thermal time scale, and it has been shown (Goldsmith, 1969) that this leads to a transition to stable phases in about $10^{6} \mathrm{yr}$.

We now consider an alternative model proposed by Parker (1966). Parker (1968b, 1969) gives references to the many papers by him and Lerche on this model. In 1960 Hoyle and Ireland pointed out that differential rotation of the Galaxy will double the disk magnetic field in about $4 \times 10^{8} \mathrm{yr}$. Since $B^{2} /(8 \pi \varrho z)$ is comparable with $K_{z}$, the support of the gas against gravitation is partly due to magnetic forces, and the increase of $B$ will lead to an increase in $z$. They argued that this process will not be uniform but will proceed fastest where the density is lowest. Further, the process is unstable (Hoyle and Harwit, 1962); since the gas will run from the elevated regions into the depressed regions to form clouds, the elevated regions will be able to expand even faster.

Parker also shows mathematically that, even if one starts with a relatively smooth field, constant in time, and parallel to the disk, the system is unstable toward perturbations in which the field acquires a sinusoidal $z$-component. The kinetic energy of the unstable gas derives from three sources: the gravitational energy of the gas sliding down the line of force, the magnetic energy released by the upwelling field lines, and the cosmic-ray energy released by the expansion of the field. The effect of gas pressure depends on the sign of $(\gamma-1)$, where $\gamma=\mathrm{d}(\log p) / \mathrm{d}(\log \varrho)$ characterizes the thermal behavior of the gas. $\gamma-1<0$ implies destabilization.

Parker's results for the case $K_{z}=$ constant are easiest to relate, and he showed that they are approximately correct for the more realistic case in which $K_{z} \propto z$. He finds the following instability criterion:

$$
\left[\frac{1}{2} \alpha+\beta+(\alpha+\beta)^{2}\right]-\left[(\gamma-1)\left(1+\frac{3}{2} \alpha+\beta\right)\right]-2 \alpha \gamma k^{2} L^{2}>0,
$$

where $\alpha=$ magnetic pressure/gas pressure, $\beta=$ cosmic-ray pressure/gas pressure, $\gamma=$ $\mathrm{d}(\log p) / \mathrm{d}(\log \varrho), L=$ scale height $=(\mathrm{d} p / \mathrm{d} \varrho)(1+\alpha+\beta) /\left|K_{z}\right|$, and $k=$ horizontal wave number of the disturbance along the magnetic field. The first term shows that magnetic and cosmic-ray pressures are always destabilizing. The second shows that gas pressure is also destabilizing if $\gamma<1$; otherwise it is stabilizing, because a hot gas will resist collapse down the field line more than a cool one. The last term shows that if the gas is thermally stable $(\gamma>0)$ short wavelengths tend to be more stable because of the restoring forces associated with strong gas-pressure gradients in short-wavelength 
disturbances. If, on the other hand, the gas is thermally unstable the effect of gas pressure is destabilizing, whatever the wavelength.

Parker has discussed a model in which $\alpha=\beta$ (equipartition between cosmic rays and magnetic field, $\beta \approx 3.5 \mu \mathrm{G}$ ). This model may be close to reality because Anand et al. (1968) have demonstrated that proper accounting for solar modulation of energetic electrons lowers the field estimated from the synchrotron emission of the Galaxy to $6 \mu \mathrm{G}$ (from the old value, $20 \mu \mathrm{G}$ ), in harmony with the values 3 to $6 \mu \mathrm{G}$ from Zeeman and Faraday rotation measurements. Equation (1) then reveals that the medium is unstable for the conditions shown in Table III. Here $k_{c}$ is a critical wave number

\section{TABLE III}

Conditions for Parker instability

$\left(K_{z}=\right.$ const., cosmic-ray $=$ magnetic pressure $)$

$\gamma=\mathrm{d} \log p / \mathrm{d} \log \varrho$
$\gamma \leqslant 0$
$0<\gamma<1$
$1<\gamma$

$\begin{array}{ll}\alpha & k \\ \text { All } & \text { All } \\ \text { All } & k<k_{c} \\ \alpha>\frac{2}{3}(\gamma-1) & k<k_{c}\end{array}$

obtained by replacing in Equation (1) the sign $>$ by $=$. We may apply to Parker's model the calculation of pressure given previously, using the results of FGH, SS, and HGG as an example. These curves are consistent with various observations to a factor of 2 , provided one takes $B \approx 3 \mu \mathrm{G}$ in the intercloud medium. From Figure 3 we see that $\gamma>0$ above $n=0.8$ nuclei $\mathrm{cm}^{-3}\left(n_{\mathrm{H}}=0.7 \mathrm{~cm}^{-3}\right.$ when 10 percent helium is taken into account). Since this is close to the observed mean density of the gas, one might consider a model in which all the gas is in the stable cool phase, close to the pressure minimum, with magnetic pressure $\left(5 \times 10^{-13}\right.$ dyne $\left.\mathrm{cm}^{-2}\right)$ much greater than gas pressure $\left(1.3 \times 10^{-14}\right.$ dyne $\left.\mathrm{cm}^{-2}\right)$. This would correspond to $\alpha=40$. Such a disk can be supported in equilibrium by the pressure of magnetic field and cosmic rays, as Parker showed, but Table III shows that the disk is unstable for all wavelengths $\lambda>\lambda_{c} \approx 2 \pi L \sqrt{ }(2 \alpha \gamma) /(1+2 \alpha)$. The value of $\gamma$ is quite sensitive to the exact value of $n$, but if we take $\gamma=0.1$ as an example, $\lambda_{c}=33$ pc.

Parker analyzed the situation in which $\gamma$ is constant. However, as the density changes the pressure will change in a much more complicated way. In fact, since the thermal relaxation time scale is short $\left(10^{5}\right.$ to $\left.10^{6} \mathrm{yr}\right)$ compared to the dynamical time scale $\left(10^{7} \mathrm{yr}\right)$, the gas will either remain close to a stable phase, or, if led into an unstable regime, make a rapid phase transition out of it. While no detailed calculations have been done, one can imagine the following sequence of events as the dynamical instability develops.

Suppose we start with a horizontal flux tube containing a uniform gas in conditions close to that of the pressure minimum in Figure 3. If the flux tube begins to develop toward a configuration like that in Figure 5, its volume will expand. Since the gas cannot leave the tube, the mean density has to drop. This drop will bring the gas in the 


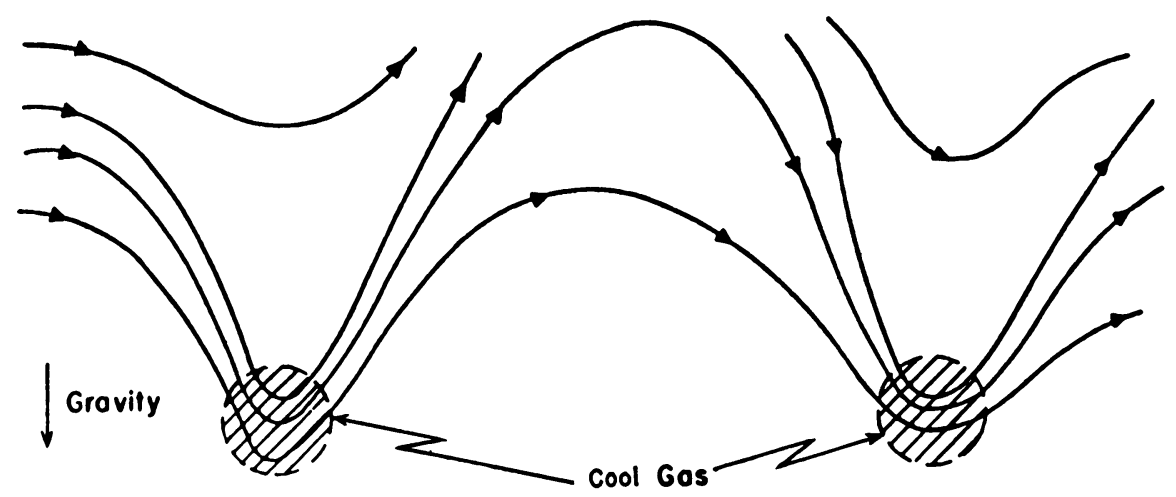

Fig. 5. Sketch of the local state of the lines of force of the interstellar magnetic field and interstellar gas-cloud configuration resulting from the intrinsic instability of a large-scale field along the galactic disk or arm when confined by the weight of the gas (Parker, 1966).

unstable phase. One may suppose that some part of the gas will expand and some part will contract. The contracting gas loses buoyancy and runs down the flux tube. In the expanding gas thermal instability develops rapidly $\left(10^{6} \mathrm{yr}\right)$. Regions less dense than average (presumably those near the top of the flux tube) are below the equilibrium line of Figure 3, and gain heat from the cosmic rays faster than they can radiate it. This increases the pressure and the rarefied gas expands. If one assumes that the gas, which started contracting at the beginning of the expansion occupied about half the volume of the flux tube the density in the hot gas can only fall by a factor of about 2 . The hot gas will therefore continue to increase in pressure, and stabilize only when it has reached the hot stable phase at $n \approx 0.2 \mathrm{~cm}^{-3}, p \approx p_{c}=2.5 \times 10^{-13} \mathrm{dyne} \mathrm{cm}^{-2}$. As the flux tube continues to rise upward, hydrostatic equilibrium gives $p=p_{0} \exp (-z / L)$ in the approximation $K_{z}=$ constant. Here $L\left[=(\mathrm{d} p / \mathrm{d} \varrho) / K_{z} \simeq 100 \mathrm{pc}\right]$ is the scale height in the hot stable phase. It seems likely that $p_{0}$, the pressure at the bottom of the field line, remains close to $p_{c}$. This pressure, according to Figure 3, requires $n=100 \mathrm{~cm}^{-3}$, $T=18 \mathrm{~K}$. While some clouds do have such characteristics, notably those that stand out in 21-cm absorption observations, most clouds are probably hotter and less dense, say $120 \mathrm{~K}$ and $15 \mathrm{~cm}^{-3}$. FGH showed that depletion of cooling elements by accretion on grains can account for the increased temperature. A theory of depletion by preferential sticking of charged species with high binding energy to a dielectric grain shows that this takes about $10^{8} \mathrm{yr}$ in a cloud having densities of the order of $10 \mathrm{~cm}^{-3}$ (Field and Zachariades, 1969).

The final appearance of the system is as shown in Figure 5 (Parker, 1966). The cloud is supported against galactic gravitation by magnetic stresses much as are solar filaments in the theory of Kippenhahn and Schlüter (1957). The detailed magnetic configuration within the cloud is of interest. Pikel'ner (1967) argued that the curvature of the field lines is small, the radius of curvature being some $150 \mathrm{pc}$. However, if his argument is modified for smaller $B$ and larger density, we obtain a radius of curvature of only a few pc, and this seems too small for the following reason. The scale height of 
the cool stable phase in the galactic gravitational field at $z=100 \mathrm{pc}$ is only $1 \mathrm{pc}$, and in the $10 \mathrm{pc}$ radius of a cloud, one would encounter a $\Delta z$ of about 3 scale heights. The intercloud pressure would then be only $1.2 \times 10^{-14}$ dyne $\mathrm{cm}^{-2}, n_{\mathrm{H}}<10^{-2} \mathrm{~cm}^{-3}$, and $n_{e}<5 \times 10^{-3} \mathrm{~cm}^{-3}$. There would be significant disagreement with pulsar observations $\left(n_{e}=2 \times 10^{-2} \mathrm{~cm}^{-3}\right)$ and observations of hot $\mathrm{HI}$ regions $\left(n_{\mathrm{H}} \approx 0.2 \mathrm{~cm}^{-3}\right)$ which agree with a model in which $p \approx p_{c}$.

Finally, we note that the presence of regimes where thermal instability occurs adds to the Parker instability by making $\gamma<0$ in Equation (1). In the final state, however, if one has only stable phases, the effect of gas pressure is stabilizing. Not only is $\gamma \approx 1$ in both phases but also the gas pressure is no longer completely trivial: $\alpha=2$ rather than 40. In Equation (1) this leads to $\lambda_{c}=300 \mathrm{pc}$, which is considerably larger than the mean length of a field line between clouds $(\approx 120 \mathrm{pc})$; so the intercloud medium is stable against further activity.

Instreaming of gas has been observed at both poles of the Galaxy (Dieter, 1969). Dieter (1964) has suggested that this could be cool material condensing from hot gas at high altitude, perhaps by thermal instability. Within the framework of Parker's theory, such gas would stream down along the lines of force looping out of the disk, much like 'coronal rain' in the solar atmosphere. If the gas is much heavier than its surroundings, it will acquire free-fall velocity equal to $10,20,30$, and $50 \mathrm{~km} \mathrm{sec}^{-1}$ for origin at $100,200,400$, and $1000 \mathrm{pc}$, respectively. As these velocities are of the order of those observed at the pole, the mechanism deserves further attention (Heiles, 1967b). The relative gas density $n(z) / n(0)$ is $0.6,0.15$, and 0.004 at $z=100,200$, and $400 \mathrm{pc}$, respectively, and is negligible at larger $z$ even if $T$ is as high as $T_{c}=9000 \mathrm{~K}$. Two mechanisms could initiate the infall. Parker (1965) has argued that the slow build-up of cosmic-ray pressure due to constant injection can initiate a local rapid expansion of the field. This could cause the relative density at the top of the field line to drop so low $(\approx 0.05)$ that a stable thermal equilibrium is impossible, and thermal instability commences. Alternatively, a finite amplitude disturbance such as a shock wave could propagate along the field line from a supernova, for example. In this case Goldsmith (1969) has shown that a phase transition can be induced (essentially because $p>p_{c}$ ), with the result that clumps of cool gas fall down the field line as required. Such mechanisms might account for activity originating up to $400 \mathrm{pc}$ altitude $(v=30 \mathrm{~km}$ $\left.\mathrm{sec}^{-1}\right)$, but it seems unlikely that activity at $1000 \mathrm{pc}\left(50 \mathrm{~km} \mathrm{sec}^{-1}\right)$ would be observable because of the negligible density there on the present model. Of course a hot corona (Spitzer, 1956) would provide the density, but at present there are doubts about the stability of a phase at $10^{6} \mathrm{~K}$. In the present picture, gas is not easily visible as it rises because it does so very slowly; it becomes visible only when descending at measurable velocities.

\section{Shock Waves and Cloud Collisions}

Kahn (1955) proposed that clouds are heated by mutual supersonic collisions. Various authors have treated the cooling of clouds subsequent to such heating, with the sim- 
plifying assumption that the gas density is constant. The results depend on certain parameters appropriate for the precise cooling mechanisms used, notably on the density of $\mathrm{H}_{2}$; values of the cooling time vary from $10^{5}$ to $10^{7} \mathrm{yr}$. If the cooling time is of the same order as the estimated time between successive cloud collisions $\left(10^{7} \mathrm{yr}\right)$, such collisions would be helpful in maintaining the high temperatures $(>100 \mathrm{~K})$ observed for some clouds.

Zimmermann $(1967,1968 \mathrm{a}, \mathrm{b})$ described the dynamics of cloud collisions in more detail, neglecting the effects of magnetic field and cosmic rays. First, two clouds come together at velocities $\approx 10 \mathrm{~km} \mathrm{sec}^{-1}$. Detailed study was made of the central collision of two spherical clouds with a relative velocity of $18 \mathrm{~km} \mathrm{sec}^{-1}$. The initial parameters were $n_{\mathrm{H}}=10 \mathrm{~cm}^{-3}$ and $T=100 \mathrm{~K}$. Second, upon first contact, a shock wave forms and proceeds into each cloud. The conditions immediately behind the shock are adiabatic, and the temperature rises to $3300 \mathrm{~K}$. Third, after a certain time has elapsed, the gas sufficiently far behind the shock has cooled down to $20 \mathrm{~K}$. The density in the cool regions rises in order to keep the pressure constant behind the shock. Fourth, the shock reaches the outer boundary of the cloud. At this point, all the gas in the two clouds has been compressed into a thin cloud of high density. The compression occurs along the line of collision, motion in the transverse dimension being neglected. The gas at the edge of the cloud begins to expand, and an expansion wave proceeds into the cloud at about the speed of sound $\left(1 \mathrm{~km} \mathrm{sec}^{-1}\right)$. This expan-

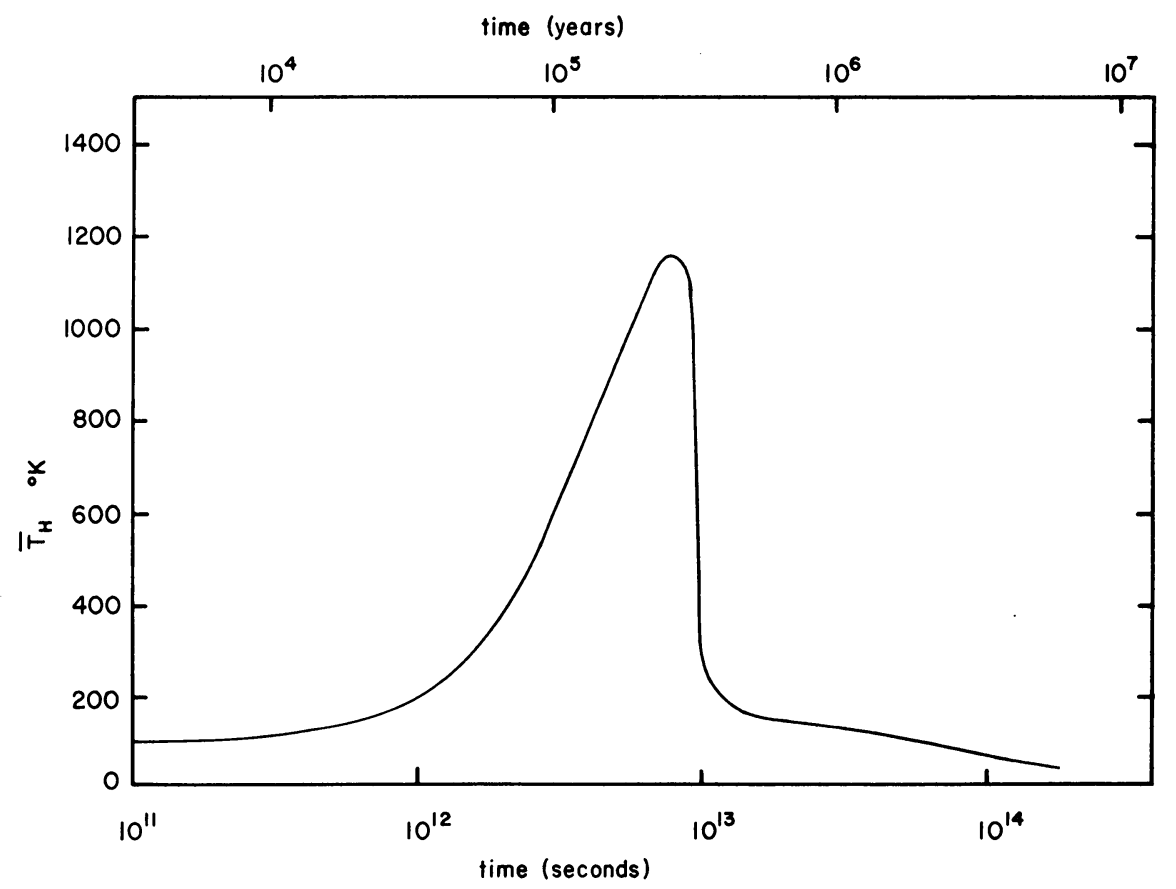

Fig. 6. The arithmetic mean temperature as a function of time of an $\mathrm{HI}$ cloud after it starts colliding with another cloud (Zimmermann, 1968b). 
sion proceeds until, fifth, the pressure in the cloud has dropped to the pressure in the intercloud medium. This requires an expansion of about a factor 15 if the expansion is adiabatic (a doubtful assumption); correspondingly, the gas temperature falls to $3 \mathrm{~K}$. Cosmic-ray heating, which would increase the temperature obtained during the expansion phase, was neglected.

Figure 6 shows the mean temperature of the whole cloud (solid curve) as a function of time. One sees first the increase in temperature as more of the cloud has gone through the shock, and then the rapid drop as the shock reaches the opposite side and cooling is completed. Since a large fraction of the cloud is at a very low temperature in the postshock region, the results would presumably be quite different if the harmonic mean temperature were shown in Figure 6, instead of the arithmetic mean temperature. Such a curve would be useful for comparing with $21-\mathrm{cm}$ measurements.

Steady shocks in Hi clouds were studied by Wentzel (1967) and by Field et al. (1968), including the effect of a magnetic field parallel to the shock front. Neither paper considered the expansion phase described by Zimmermann. Field et al. included a variety of cooling mechanisms; in particular, fine-structure excitation of $\mathrm{O}_{\mathrm{I}}$ by hydrogen atoms, and excitation of the fine-structure levels of $\mathrm{C}^{+}, \mathrm{Si}^{+}$, and $\mathrm{Fe}^{+}$by collisions with hydrogen atoms, in addition to those considered by Zimmermann. Figure 7 summarizes the results of a number of calculations of cooling time (defined as the time for the gas to drop back to $100 \mathrm{~K}$ from the postshock temperatures) for various shock velocities and magnetic field strengths. This figure is based on $\mathrm{H}_{2} / \mathrm{H}=10^{-3}$ but the results for $\mathrm{H}_{2} / \mathrm{H}=10^{-5}$ are very similar; the latter value is probably more

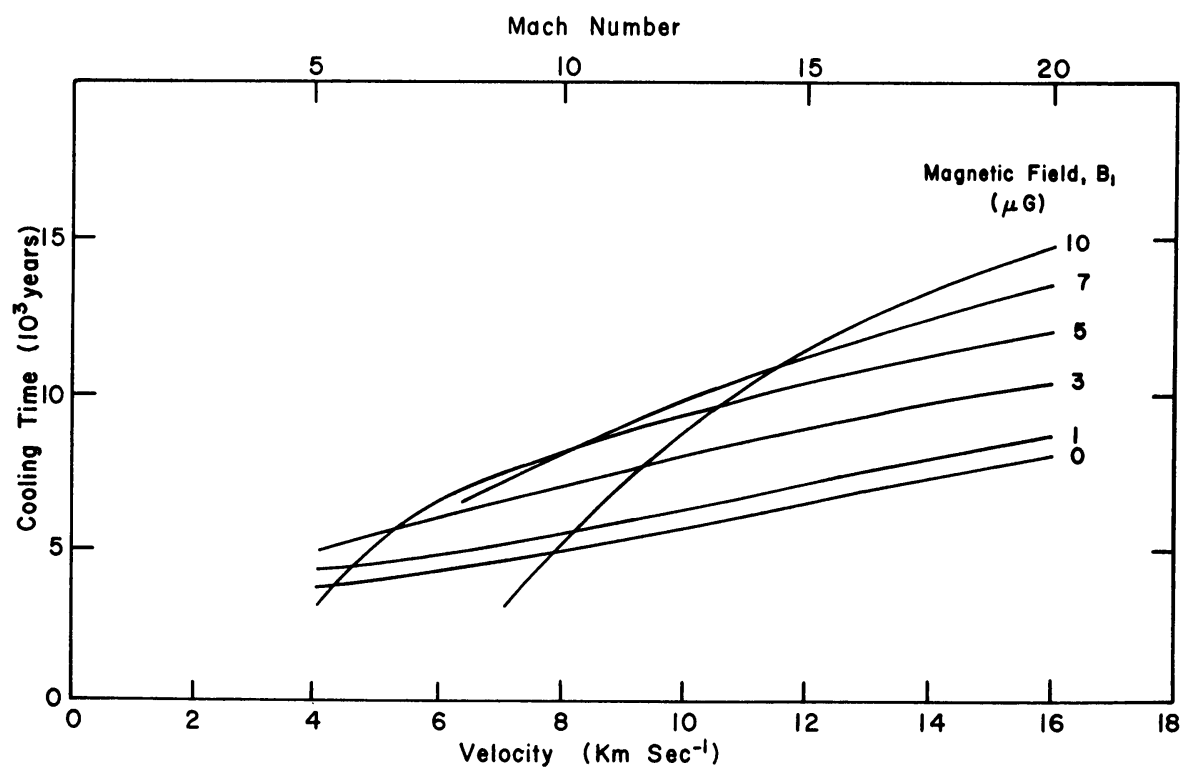

Fig. 7. Cooling time for $\mathrm{HI}$ gas after passage through a shock front. The magnetic field is parallel to the front. $\mathrm{H}_{2} / \mathrm{H}=10^{-3}$, but a much lower value would give approximately the same results (Field et al., 1968). 
realistic (Stecher and Williams, 1967). The magnetic field has two compensating effects on the cooling time: first a decrease in time by reduction of the original postshock temperature, and second, an increase in time by prevention of a density rise in the cool regions. As a result, the cooling times are relatively insensitive to the value of $B$ over the plausible range 0 to $10 \mu \mathrm{G}$. It appears that for shock velocities to be encountered in cloud collisions (half the relative velocity, about $10 \mathrm{~km} \mathrm{sec}^{-1}$ ) the cooling time should be less than $10^{4} \mathrm{yr}$ - a significant reduction from the $10^{6} \mathrm{yr}$ found by Zimmermann for the case $\mathrm{H}_{2} / \mathrm{H}=0$. The difference is attributed to his neglect of $\mathrm{O}_{\mathrm{I}}$ cooling, which dominates in every case.

If we could be confident of the present results, we could state definitely that cloud collisions cannot maintain cloud temperatures at the observed values, since $10^{4} \mathrm{yr}$ is such a small fraction of the time it takes $\left(10^{6} \mathrm{yr}\right)$ for a $10 \mathrm{~km} \mathrm{sec}^{-1}$ shock to cross a $10 \mathrm{pc}$ cloud. After the first $10^{4} \mathrm{yr}$ has elapsed, a cloud collision actually decreases the cloud temperature by compressing a large fraction of the cloud to such a high density that cosmic rays cannot keep it hot.

Unfortunately, serious revisions are required before we can be certain of this:

(i) The cross section for excitation of $\mathrm{O}$ by $\mathrm{H}$ should be reduced a factor 3 (Bahcall and Wolf, 1968).

(ii) The free electrons from cosmic-ray ionization should be included in electronion cooling.

(iii) Account should be taken of depletion on grains.

(iv) The excitation of the $a^{4} F$ term of $\mathrm{Fe}^{+}$should be included (Pottasch, private communication).

Of these, (i) and (iii) will increase $T$ and (ii) and (iv) will decrease it, so the outcome is hard to predict. In addition to these revisions, study should be directed to the effect of an oblique magnetic field, the coupling of the magnetic field to the neutrals through the ions (Wentzel, private communication) and the dynamical effects of cosmic-ray pressure (Hudson, 1965, 1968).

A more general problem is whether cloud collisions actually occur as described. As Pikel'ner (1967) points out, the simple concept of a cloud collision will be modified by the presence of a magnetic field. He estimates that a typical cloud will oscillate perpendicular to the field about its mean position with an amplitude of 10 to $15 \mathrm{pc}$. Since this distance is considerably less than the mean free path for collisions, the number of collisions will be reduced. Furthermore, collisions perpendicular to the field will tend to be elastic as shown by Pikel'ner (1957) and Kogure (1965); we have mentioned evidence of this above, in the reduced heating by shock waves normal to the field. In summary, collisions across the field will be relatively ineffective in heating the gas.

Pikel'ner has argued that motions along the field are unimpeded, because the depth of the wells in which they settle is small - only a few pc - so that little displacement of the lines of force is necessary for motion to occur. This agrees with the observational fact that random cloud motions seem to occur equally parallel and perpendicular to the field. At first sight it might appear that deep wells (such as might occur in the 
Parker model) would keep gas from moving along the field. It is true that energy must be put into the expanded magnetic field in front of the cloud to compress it to the value within the cloud. However, as much of this energy is regained again upon emerging from the rear of the cloud, the net loss of kinetic energy is small. We note that the speed of the cloud is of the same order $\left(\approx 10 \mathrm{~km} \mathrm{sec}^{-1}\right)$ as the sound speed in the intercloud medium, so there is probably little dissipation by a shock wave there. We tentatively conclude that collisions along the field can occur in a one-dimensional way, although much analysis remains to be done here.

If the collision event takes place only between clouds (or parts of clouds) on the same lines of force, transverse motion is inhibited, and the description of Zimmermann is quite accurate. While the speed of cooling behind the shocks is still uncertain, we can be confident that it is somewhat faster than the collision itself, so that collisions along the lines of force are quite inelastic. Each cloud therefore grows in mass, and the trend toward larger clouds will continue until some other process intervenes. According to Spitzer (1968a, p. 221), gravitational collapse for masses larger than the Ebert-Bonnor critical mass is one such process. For a pressure in the intercloud medium $p=2.5 \times 10^{-13}$ dyne $\mathrm{cm}^{-2}(n T=1800)$, this critical mass is $\approx 10^{3} M_{\odot}$; Spitzer argues, on the basis of the virial theorem (and more detailed arguments by Field, 1969, confirm this), that a field of $3 \mu \mathrm{G}$ will increase this critical mass only by a factor of 2 or 3. Presumably fragmentation and star formation is the normal outcome of this process. According to Oort (1954), after formation, the more massive stars will create HII regions in the remaining gas of the cloud and accelerate it outward, accounting for the kinetic energy retained by the clouds in spite of their inelastic collisions. We shall return to the problem of $\mathrm{H}$ II regions below, but here we note that in the Parker model, all this would occur in a massive cloud at the bottom of a magnetic well, and kinetic energy would preferentially be transmitted by clouds thrown out along the field.

Field and Saslaw (1965), Field and Hutchins (1968), and Penston et al. (1969) proposed and developed a statistical model of the cloud mass spectrum, assuming that it is fed by small units from expanding Hil regions and is drained by collapse of massive clouds. They found a quasi-equilibrium spectrum $M^{-3 / 2}$, which is in fair agreement with observation. The latter paper shows that under certain reasonable assumptions the velocity distribution of clouds should obey equipartition and be Maxwellian. All of this work should be revised to take account of additional phenomena, such as the dynamical interactions with intercloud gas, the generation of new clouds by instabilities, and the constraint of one-dimensional motion imposed by the magnetic field.

All of the work on cloud collisions suffers from inadequate contact with observation. While radio astronomers have given us much data on mean values of temperatures, density, and magnetic field, I am not aware of observations of an individual cloud collision or even an individual shock wave (in the $21-\mathrm{cm}$ line). It is dangerous to proceed with theoretical models without such observational checks. Field et al. (1968) estimated the fluxes of infrared lines between 4 and $156 \mu$, which would be emitted by 
the post-shock gas, and concluded that these are marginally observable with advanced equipment. Pottasch (1968) and Osterbrock (1969) have performed similar calculations for $\mathrm{HI}$ gas under other conditions. If such lines can be observed, information about temperature, density, composition, and spatial configuration can be achieved. Since the emission should be particularly intense from shock waves and other regions where dissipation is occurring, infrared measurements should tell us about the smallscale dynamics of the medium.

\section{HII Regions; Expansion and Inhomogeneities}

It has long been realized that the high temperature of $\mathrm{HII}$ regions ( $\$ 10000 \mathrm{~K}$ ) and a similar density with respect to neighboring HI regions provide a pressure gradient which may be dynamically important. In the years since the last Symposium, considerable calculation has clarified this situation.

Spitzer and Savedoff (1950) calculated equilibrium temperatures of HII regions, balancing the gains by photoionization of hydrogen against collisional losses due to excitation of both fine-structure and optical levels of impurity atoms. They obtained values in the range ( 5 to 13$) \times 10^{3} \mathrm{~K}$ and pointed out that the observed strength in many nebulae of $\mathrm{O}^{+} \lambda 3727(E / k=39000 \mathrm{~K})$ requires temperatures of this order. Burbidge et al. (1963) revised these calculations, emphasizing the importance of finestructure transitions such as ${ }^{2} P_{3 / 2}{ }^{2} P_{1 / 2}$ of $\mathrm{Ne}^{+}$at $12.8 \mu$. Taking $\mathrm{Ne} / \mathrm{H}=5 \times 10^{-4}$ and $\Omega=1$ (est.), they found $T=3500 \mathrm{~K}$ if the central star has a $T$ of $30000 \mathrm{~K}$. Osterbrock (1965) showed that $\Omega$ was only 0.3 , so that $T$ increased to $6000 \mathrm{~K}$ for the same type of star. He also called attention to the fact that collisional de-excitation of $\mathrm{O}^{++}$, $\mathrm{N}^{+}$, and $\mathrm{C}^{+}$for $n_{e}>10^{4} \mathrm{~cm}^{-3}$ reduces the cooling rate. Hjellming $(1966,1968)$ did a complete calculation, taking into account the detailed ionization equilibrium of the elements responsible for cooling, the transfer of Lyman continuum radiation, the hardening of the radiation by absorption as it proceeds through the nebula, the effects of collisional de-excitation, and the detailed flux distribution of the stellar atmosphere. He used modern $\Omega$ 's (Saraph et al., 1969). This work was extended to non-uniform densities by Rubin (1968). For a main-sequence star of $30000 \mathrm{~K}$ Hjellming obtained $5800 \mathrm{~K}$ for a low-density region near the star. This temperature drops by about $800 \mathrm{~K}$ in the outer parts of the nebulae because of the Lyman-continuum diffuse radiation field. If one raises $n_{e}$ to $\sim 10^{4} \mathrm{~cm}^{-3}$ then $T$ will go up by about $2000 \mathrm{~K}$, whereas if one increases the optical depth $\tau$ at the Lyman limit to $2, T$ goes up by about $1000 \mathrm{~K}$. Hjellming concludes that $6000 \mathrm{~K}$ is a typical temperature, with values up to $9000 \mathrm{~K}$ being possible in dense or optically thick regions, and values down to $4000 \mathrm{~K}$ in lowdensity outer regions. Inspection of his results indicates that fine-structure transitions often play only a minor role. This would be especially true if the abundance of neon is only $6 \times 10^{-5}$ (Flower, 1969; Pagel, 1969) rather than $4 \times 10^{-4}$ as he assumed. We note that in addition to the temperature equilibrium calculations described here, Hjellming computed the structure of various types of ionization fronts.

There is some controversy as to whether theory and observations yield different 
temperatures. Peimbert (1967) gives optical results for three nebulae, based on the ratios of auroral-to-nebular lines of heavy ions and line-to-continuum intensities of hydrogen. He obtains values between 6000 and $14000 \mathrm{~K}$ in the center of Orion, depending on the method. Kaler (1968) reviews other optical data with similar results. Measurements based on the ratio of radio recombination lines to free-free continuum seem to give systematically lower values (Dieter, 1967; Mezger, 1968) but are unreliable because of non-LTE effects. Radio measurements based on fitting the shape of the free-free continuum give very low values in two cases (Terzian et al., 1968). These authors obtained $3000(+2000 ;-1000) \mathrm{K}$ for Orion. Since the method is sensitive primarily to the temperature of the outer parts, they suggest that the discrepancy between optical and radio measurements reflects a strong inward temperature gradient. This may be in qualitative agreement with the calculations of Hjellming (1968), because the diffuse radiation field tends to decrease $T$ in the outer parts, while collisional de-excitation tends to increase it in the dense inner parts.

One of the aspects of $\mathrm{HII}$ regions of interest here is the dynamical expansion caused by high thermal pressure. The above discussion indicates that the theoretical situation

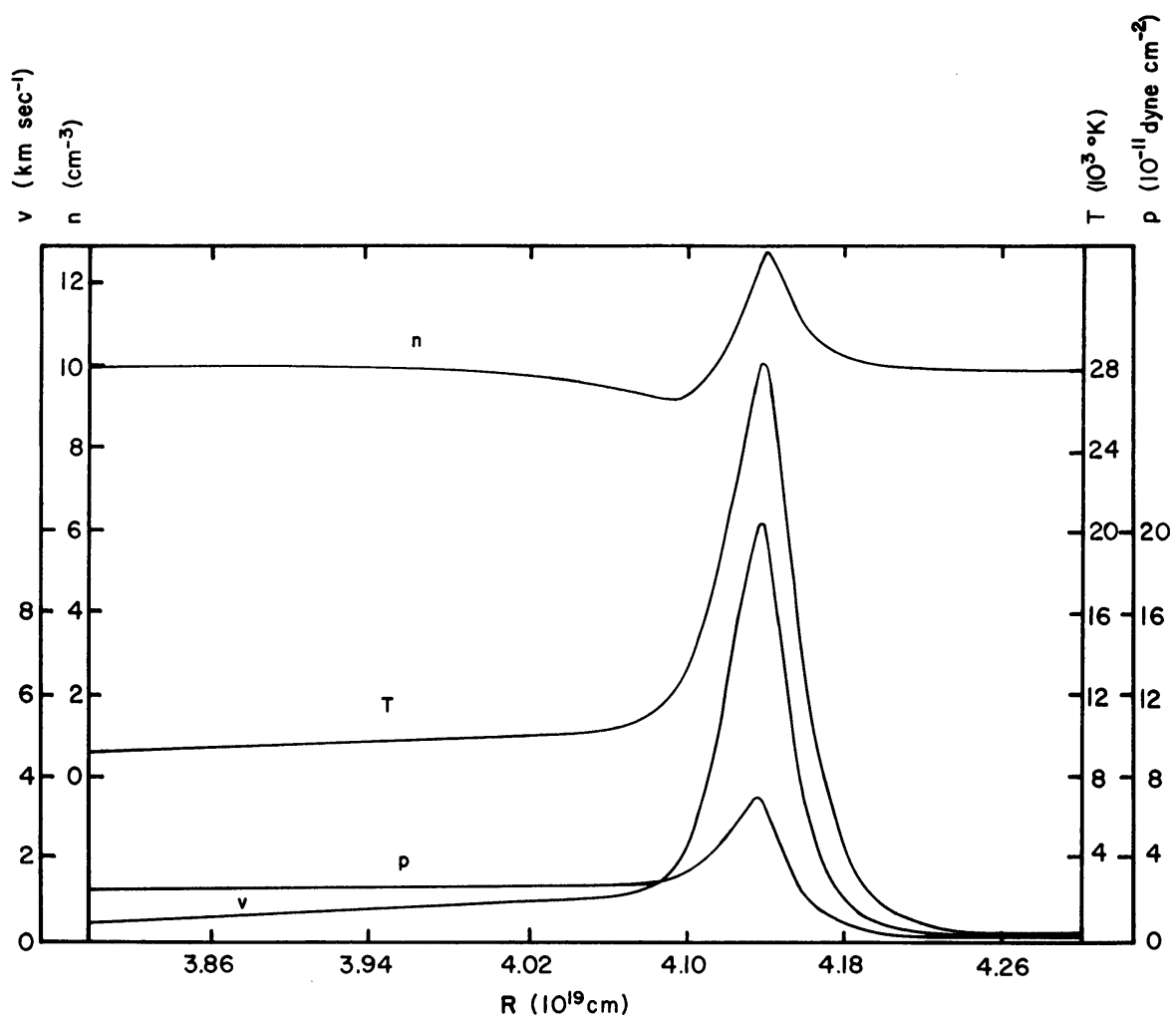

Fig. 8. Variation of velocity $v$, number density $n$, temperature $T$, and pressure $p$ as a function of radius $R$ from the star in the vicinity of an ionization front moving away from an $\mathrm{O}$ star with $T=42000 \mathrm{~K}$ after $3 \times 10^{4} \mathrm{yr}$ (Mathews, 1965). 


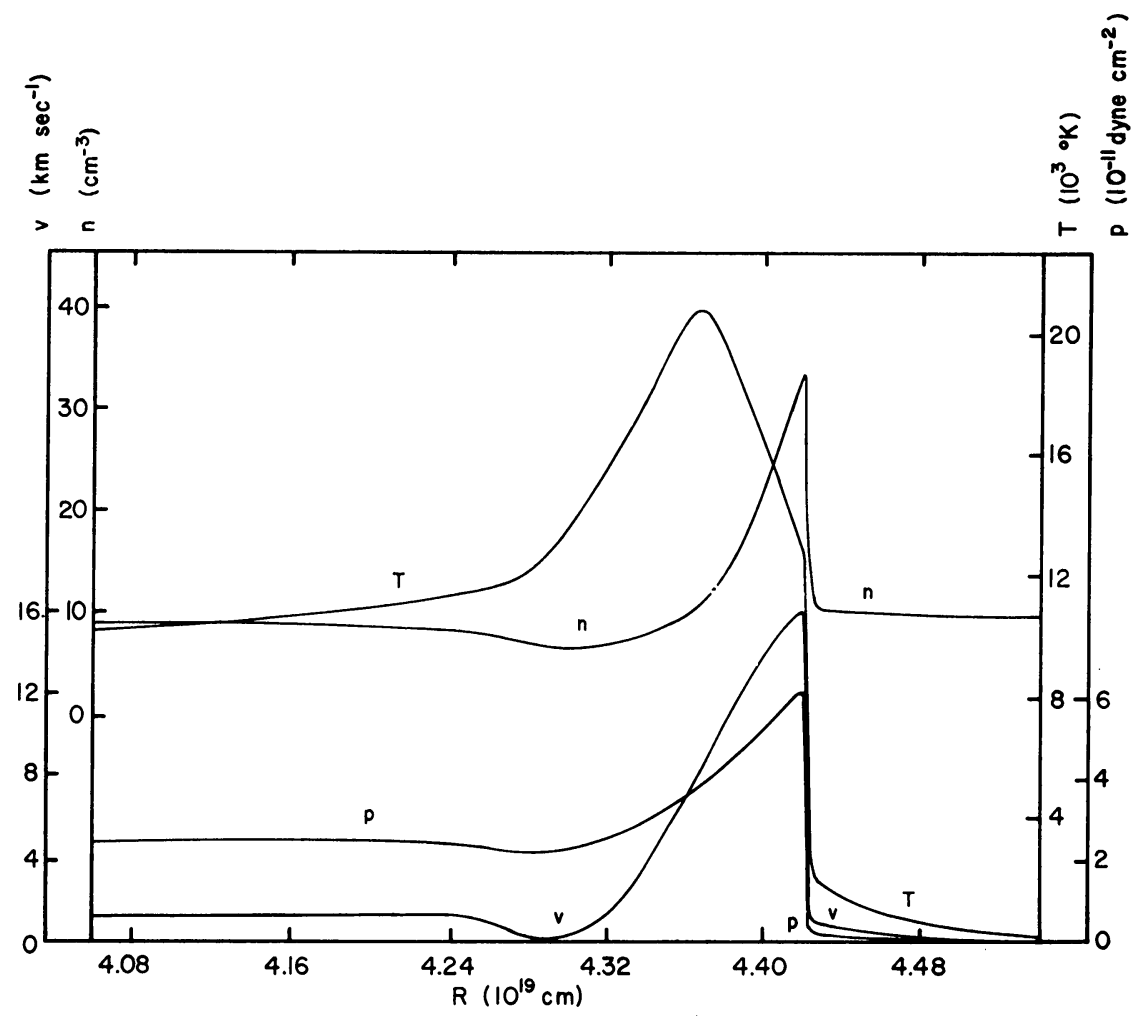

Fig. 9. The same as in Figure 8, but after $6 \times 10^{4} \mathrm{yr}$.

regarding the temperature is well in hand and that there are no glaring disagreements with observation. We now turn to two recent discussions of the expansion problem, by Mathews (1965) and Lasker (1966a, b). (A review of these and early studies of the problem has been given by Mathews and O'Dell, 1969). Earlier work on this problem had been restricted to similarity solutions but the papers of Mathews and of Lasker are based on numerical solution of the partial differential equations involved for specified initial values and use finite-difference methods.

Mathews (1965) treats the evolution of an HI region with $n_{\mathrm{H}}=10 \mathrm{~cm}^{-3}$ and $T=$ $100 \mathrm{~K}$ as it is ionized by a $30 M_{\odot}$ star, whose main-sequence temperature is $42000 \mathrm{~K}$. Mathews treats in detail the luminosity of the star during its approach to the main sequence, since the time of approach $(6000 \mathrm{yr})$ is comparable to $1.3 / \alpha n=10^{4} \mathrm{yr}$, the classical time of formation of the Hil region. As predicted from earlier work, an R-type ionization front moves very rapidly into the gas, averaging $1000 \mathrm{~km} \mathrm{sec}^{-1}$ in the first $10^{4} \mathrm{yr}$. Velocities of the ionized gas remain less than $0.2 \mathrm{~km} \mathrm{sec}^{-1}$ during this phase, in accordance with prediction for steady R-type fronts. At $6 \times 10^{4} \mathrm{yr}$, the buildup of the pressure wave behind the ionization front has developed into a shock front which leads the ionization front by 3 per cent of the radius. Although the com- 
putations were terminated at this point, the much-discussed detachment of a shock front which permits the ionization front to be D-type by virtue of its compression of the neutral hydrogen was demonstrated by Mathews (Figures 8 and 9).

Lasker (1966a) assumed that his star starts to shine instantaneously; his initial conditions are similar to those of Mathews. Unlike Mathews, who followed the Lyman-continuum radiation field in detail, he approximates the ionization front by a discontinuity through which atoms flow and are instantly ionized and heated to $10000 \mathrm{~K}$ by the ultraviolet photons which escape absorption in the Hil region; hence his results are unreliable in the immediate vicinity of the ionization front. On the other hand, in certain models, approximate allowance is made for cooling behind the shock (in the $\mathrm{HI}$ ). His computations extend up to $2 \times 10^{6} \mathrm{yr}$, much longer than those of Mathews, and one can therefore examine the later stages of development which are probably more pertinent to observed Hil regions. Figure 10 shows his results for a case with no HI cooling. One sees the detachment of the shock at about $8 \times 10^{4} \mathrm{yr}$, and the progressive acceleration of ever-increasing amounts of $\mathrm{HI}$ as time goes on. At about $9 \times 10^{5} \mathrm{yr}$ an expansion wave caused by the acceleration of the outer $\mathrm{HII}$ has travelled to the center; thereafter the central density of $\mathrm{H}$ II decreases as the region expands.

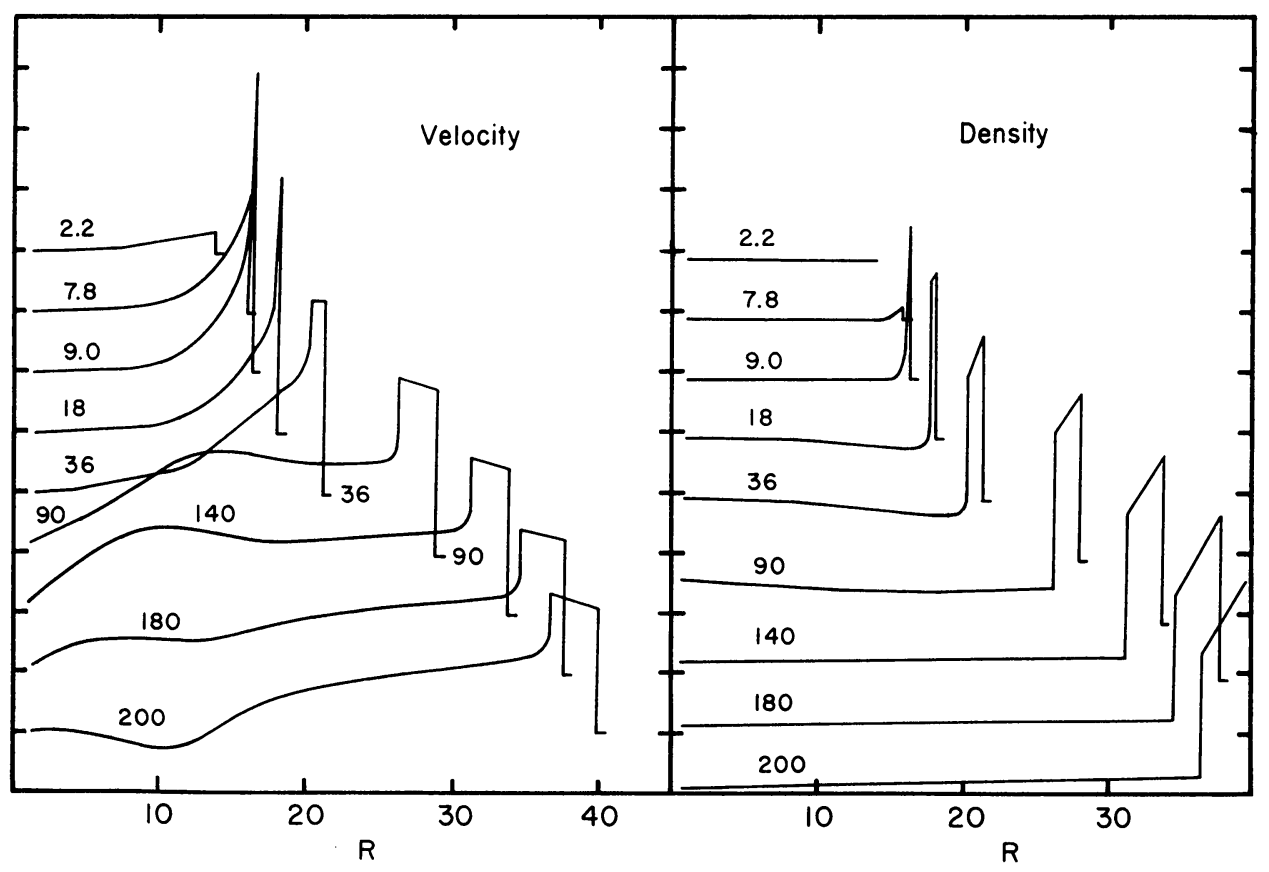

Fig. 10. Variation of velocity $V$ and density $n$ as a function of radius $R$ from the star for one of the cases of expanding $\mathrm{H}_{\mathrm{I}}$ regions considered by Lasker (1966a). To each curve is added the time after beginning of the expansion expressed in units of $10^{4} \mathrm{yr}$. The velocity unit is $3.2 \mathrm{~km} \mathrm{sec}^{-1}$, the density unit $6.4 \mathrm{~cm}^{-3}$. 
The thicknesses Lasker obtained for the Hi shock seem unrealistically small. The cooling time of $\mathrm{HI}\left(10^{4} \mathrm{yr}\right)$ is small compared to the evolution time $\left(2 \times 10^{6} \mathrm{yr}\right)$. Lasker therefore considered an additional model with an exponential cooling $e^{-t / \tau}$, with $\tau=$ $5 \times 10^{4} \mathrm{yr}$, and found that the evolution of the HII region is changed hardly at all. On the other hand, $\Delta r$, the thickness of the compressed Hi region, decreases from about 10 per cent of the radius of the shock, to only 1 per cent, with a corresponding increase in density. Apparently the I-front acts like a piston which forces a shock at about $10 \mathrm{~km} \mathrm{sec}^{-1}$ to compress the Hi up to about the same pressure as the HII. It does not matter much whether this pressure is due to a high temperature (no cooling) or a high density (cooling). In the absence of magnetic fields, however, one would expect cooling to be effective in bringing about compression, and consequently, that a very thin but dense $\mathrm{HI}_{\mathrm{I}}$ shell should be found around old $\mathrm{H}$ II regions. The available

TABLE IV

$\mathrm{H}_{\text {II }}$ regions with observed $\mathrm{HI}$ shells

\begin{tabular}{|c|c|c|c|c|c|}
\hline Nebula & $\begin{array}{l}\text { Radius of } \\
\text { I-front } \\
\text { (pc) }\end{array}$ & $\begin{array}{l}\text { Outer radius } \\
\text { (pc) }\end{array}$ & $\Delta R / R$ & $\begin{array}{l}\text { Expansion } \\
\text { velocity } \\
\left(\mathrm{km} \mathrm{sec}^{-1}\right)\end{array}$ & References \\
\hline Rosette & 20 & 50 & 0.6 & 15 & Raimond, 1966 \\
\hline NGC 6910 & 1.5 & 5.5 & 0.7 & 10 & $\begin{array}{l}\text { Davies and } \\
\text { Tovmassian, } 1963\end{array}$ \\
\hline$\lambda$ Ori & 13 & 30 & 0.6 & 8 & Wade, 1958 \\
\hline I Mon & 40 & 90 & 0.7 & 23 & $\begin{array}{l}\text { Girnstein and } \\
\text { Rohlfs, } 1964\end{array}$ \\
\hline NGC 5146 & 1.5 & 3.3 & 0.5 & $<6$ & Riegel, 1967 \\
\hline NGC 281 & 7.5 & 15 & 0.5 & $<8$ & Riegel, 1967 \\
\hline DR 21 & 0.4 & - & - & 25 & $\begin{array}{l}\text { Thompson, } \\
\text { Colvin and } \\
\text { Hughes, } 1969\end{array}$ \\
\hline
\end{tabular}

observations seem at first glance to disagree with this. In Table IV we note some $21-\mathrm{cm}$ observations of expanding $\mathrm{HI}$ shells around $\mathrm{H}$ II regions. In every case $\Delta R / R$ is much larger, not only than the value of $10^{-2}$ predicted for $\mathrm{HI}$ cooling, but also than the value of $10^{-1}$ predicted for purely adiabatic shocks. It is interesting that both $\lambda$ Ori and NGC 5146 have dust shells around them of the order of a few per cent of the radius (Wade, 1958; Riegel, 1967). Possibly this dust (which in places has several magnitudes of extinction) marks the actual compressed region. From this conclusion it would follow that the $21 \mathrm{-cm}$ antenna beams are too large to resolve the expanding shells (Lasker, 1966a; Riegel, 1967). We note that infrared observations at the wavelengths of cooling transitions should be able to locate the Hi shock unambiguously with high resolution (Field et al., 1968).

Among the mechanisms suggested for increasing the width of the compressed region are turbulent or magnetic pressures in the $\mathrm{HI}$ region, a decrease in driving pressure as the star evolves, or expansion of the outer part of the cloud into the inter- 
cloud medium. Lasker (1966b) investigated the effects of magnetic fields on I-fronts and concluded that if $Q=B^{2} / 8 \pi p \lesssim 1$ ahead of the front, there is little effect (for either R- or D-type fronts). In fact, $Q=1.5$ if $B=3 \mu \mathrm{G}$ in a cloud at the critical pressure found by Field et al. (1969), $2.5 \times 10^{-13}$ dyne $\mathrm{cm}^{-2}$. After adiabatic compression by a $10 \mathrm{~km} \mathrm{sec}^{-1}$ shock, $Q$ falls to 0.33 , so that an I-front moving into such a region is relatively unaffected. However, cooling behind the shock increases $Q$ considerably (by reducing $p$ ). Lasker (1966a) discusses this situation for the two extreme cases: (i) the angle between magnetic field and radius from the star is $0^{\circ}$ and (ii) the angle is $90^{\circ}$. Along the magnetic field (zero angle) there is no effect so the compressed H I region remains thin, while at an angle of $90^{\circ}$ simple equations shows that the compressed region is about 10 times thicker. As Lasker notes, however, at intermediate angles the thicknesses may not be intermediate, since there can be discontinuous transitions from one branch of solutions to another (corresponding to the fast and slow hydromagnetic waves) as the angle between $\mathbf{B}$ and the radius vector varies from $0^{\circ}$ to $90^{\circ}$. More work on this problem is recommended, both observationally (by seeking thin compressed regions) and theoretically (by verifying the correct branches of solutions for the shock and I-fronts in the context of the actual initial-value problem).

Lasker (1967) summarized numerical results on Hil-region evolution in a simple way. The results indicate that (i) the radius $r_{i}$ of the ionized region very quickly (about $10^{4} / n$ yr) approaches the value $s_{0}=\left(3 S / 4 \alpha n_{0}^{2}\right)^{1 / 3}$ predicted by static Strömgren theory ( $\alpha$ is the recombination coefficient, $S$ the rate of emission of ionizing photons from the exciting star), (ii) thereafter $r_{i}$ grows slowly as the I-front moves into the shocked HI, always satisfying the Strömgren relation for the actual density, $n$, in the HII region, (iii) $n$ remains rather uniform and the velocities within the HII region remain $<\frac{1}{4} c$ (the isothermal sound speed in the HII region), (iv) the pressure is constant through the I-front after the early formation period, (v) the shock front never moves far ahead of the I-front. The last fact means that $\mathrm{d} r_{i} / \mathrm{d} t \approx v_{s}$ where the shock velocity $v_{s}$ for strong shocks is given by $\left(4 p^{\prime} / 3 p_{0}\right)^{1 / 2} c_{0}$, where $p_{0}$ and $c_{0}$ refer to the unshocked HI and $p^{\prime}$ to the shocked HI. Since $p^{\prime}=p_{\mathrm{II}}=\varrho_{\mathrm{II}} c^{2}$ from (iv), where $c$ is the sound speed in the HII region, and $p_{0}=\varrho_{0} c_{0}^{2}$, it follows that $v_{s}=c_{1}\left(n / n_{0}\right)^{1 / 2}$, where $c_{1}=2 c / \sqrt{ } 3$. From (ii), $n / n_{0}=\left(r_{i} / s_{0}\right)^{-3 / 2}$, so that

$$
\mathrm{d} r_{i} / \mathrm{d} t \approx v_{s}=c_{1}\left(r_{i} / s_{0}\right)^{-3 / 4},
$$

which has the solution

$$
r_{i}=s_{0}\left(1+7 c_{1} t / 4 s_{0}\right)^{4 / 7}
$$

where $t$ is the time elapsed since the Strömgren region formed $\left(r_{i}=s_{0}\right)$. Hence

$$
n=n_{0}\left(1+7 c_{1} t / 4 s_{0}\right)^{-6 / 7} .
$$

These expressions are found to represent the numerical results quite well. The I-front can be considered to be a piston exerting a pressure $\varrho c^{2}$. As it moves, it does work on the $\mathrm{HI}$ ahead. Of the energy imparted by the strong shock, half goes into heat and 
half into kinetic energy. Hence the kinetic energy of the accelerated $\mathrm{HI}_{\mathrm{I}}$ is

$$
E_{k}=\int p \mathrm{~d} V=2 \pi \varrho_{0} c^{2} s_{0}^{3} \int_{s_{0}}^{r_{i}}\left(\frac{n}{n_{0}}\right)\left(\frac{r_{i}}{s_{0}}\right)^{2} \frac{\mathrm{d} r_{i}}{s_{0}},
$$

which from Equations (3) and (4) is equal to

$$
E_{K}=(4 \pi / 3) \varrho_{0} c^{2} s_{0}^{3}\left[\left(r_{i} / s_{0}\right)^{3 / 2}-1\right] .
$$

This is readily shown to be $\frac{2}{3}\left[1-\left(r_{i} / s_{0}\right)^{-3 / 2}\right]$ times the thermal energy in the $\mathrm{HII}$ region. It is important to compare $E_{K}$ to the energy available from ultraviolet photons, $E_{L}=S t \Delta E$, where $\Delta E$ is the average energy going into heat per ionization $\left(\Delta E \approx k T_{*}\right)$. By using the Strömgren relation one can show that

$$
\varepsilon=\text { efficiency }=\frac{E_{K}}{E_{L}}=\frac{2 k T}{\Delta E \frac{1}{\alpha n t}}\left(1-\frac{n}{n_{0}}\right) .
$$

For late phases of evolution $\left(n \ll n_{0}\right)$ this has the simple interpretation that the total number of recombinations per proton is $\alpha n t$, and since each is balanced by an ionization involving the expenditure of $\Delta E$, the total energy put into the $\mathrm{H}_{\text {II }}$ is $\alpha$ t $\Delta E$ erg per proton. Almost all of this energy from the star goes into keeping the gas hot, only the fraction $2 k T$ being the pressure (which can do work) available per proton. Lasker shows that when $t=$ main sequence lifetime of the star and $n=$ the corresponding final density starting with $n_{0}=10 \mathrm{~cm}^{-3}, \varepsilon$ varies from 0.002 for spectral type 05 to 0.07 for $B 1$. When weighted by the numbers and fluxes of stars of various spectral types, the average efficiency is $\varepsilon=0.0024$ (as O-stars supply most of the energy). A higher efficiency $\left(\varepsilon^{\prime}=0.014\right)$ is required (Spitzer, 1968a) to account for the motions of interstellar clouds by the kinetic energy of $\mathrm{HI}_{\mathrm{I}}$ shells accelerated by $\mathrm{H}$ II regions (OortSpitzer mechanism). As the discrepancy is only a factor 6 , our tentative conclusion is that a significant fraction of the energy dissipated in cloud collisions, but probably not all, originates in HII regions. The alternatives include inflation of the magnetic field with resulting downward motion of gas clouds (Hoyle and Ireland, 1960; Parker, 1966), infall of intergalactic matter (Oort, 1969), electromagnetic radiation from neutron stars (Hoyle et al., 1964), and expansion of supernova shells (Shklovskii, 1962; Kahn and Dyson, 1965; Kahn and Woltjer, 1967; Spitzer, 1968b).

Kahn and Woltjer (1967) make quantitative estimates of the efficiency of cloud acceleration by supernova shells. They adopt a snowplow model appropriate for rapid cooling of shocked gas, and consider two cases of shell speed. If the speed is relativistic, the efficiency is $\varepsilon_{R}=\frac{1}{2}(v / c)^{1 / 2}$, while for non-relativistic shells it is $\varepsilon_{N R}=v / V$, where $v=10 \mathrm{~km} \mathrm{sec}^{-1}$ is the speed of interstellar clouds and $V=7000 \mathrm{~km} \mathrm{sec}^{-1}$ is an estimated shell velocity. Hence $\varepsilon_{R}=0.003$ and $\varepsilon_{N R}=0.0015$, of the same order as found by Lasker (1967) for HII regions. Using the data for dissipation in cloud collisions used above, adopting the non-relativistic model, and taking the supernova rate as 1 per $100 \mathrm{yr}$, we find that the required kinetic energy of the shell is $3 \times 10^{51}$ 
erg, compared to the observational estimate of $10^{51}$ erg (Minkowski, 1968). H II regions contribute $\frac{1}{6}$ and supernovae contribute $\frac{1}{3}$ of what is required, so together they may well account for the motions of clouds.

When the acceleration is complete, the radius of the supernova shell is about $50 \mathrm{pc}$ according to Kahn and Woltjer (1967). Shane and Katgert (1967) have tentatively identified such a feature observationally. The large radius means that many clouds are actually members of expanding shells. From the model of Kahn and Woltjer one can estimate that the fraction is roughly 20 per cent. It is interesting that according to Spitzer (1968b), about 10 per cent of the calcium clouds observed are of relatively high velocity and show an excess of negative velocities as if they had been recently accelerated away from the vicinity of the early-type stars against which they are seen.

In closing our discussion of the expansion of Hil regions, reference is made to the effects of stellar winds. Mathews (1966) has shown that mass loss of the order $10^{-5} M_{\odot}$ $\mathrm{yr}^{-1}$ can, by the pressure it exerts on the interior of the gas, cause the observed hole at the center of the Rosette Nebula, while Pikel'ner (1968b) has constructed a model based on stellar winds to explain the high internal velocities observed in some $\mathrm{HII}$ regions (Shcheglov, 1968). Mathews (1967) has also considered the effect of radiation pressure on grains within the Hil region. He finds that the electrostatic drag on the charged grains can transmit the pressure effectively to the gas, with the result that the inner part of the Rosette Nebula can be evacuated.

We now turn to our final topic, inhomogeneities in Hil regions. There is ample evidence that such inhomogeneities exist: determination of $\left\langle n_{e}\right\rangle$ by OII $\lambda 3727$ yields lower values than determination of $\left\langle n_{e}\right\rangle^{1 / 2}$ from hydrogen emission; the forbidden lines yield higher temperatures than the hydrogen lines and the radio continuum; the emission lines are broadened by motions comparable to the thermal velocity and, in some cases, are split in local regions. According to Kahn (1967), one can possibly interpret the discrepancy between the ages of the stars in Orion and the calculated expansion age of the Nebula, if the Nebula is actually made up of many dense globules of neutral matter which are being slowly ionized at present. This model can also account for the observed line splitting, by streams of ionized gas emerging from the globules and colliding to form shock waves. No doubt it would also account qualitatively for the variations in $n_{e}$ and $T$ observed, and possibly for the presence of $\mathrm{OI}_{\mathrm{I}}$ $\lambda 6300$, which has always been hard to understand in a completely ionized region.

Some divergence exists on the theoretical treatment of globules. $\mathrm{T}$ Tau stars are often seen imbedded in elephant-trunk or comet-tail structures at the edges of $\mathrm{HII}$ regions and Dibai (1963) suggested that this could be explained as the result of a converging shock wave, initiated by the high pressure in the surrounding Hir. Later Dibai and Kaplan (1964) studied such a shock wave by the method of similarity solutions and obtained velocities up to two times the speed of sound in Hil regions and density increases (after passage of first a converging and then a reflected shock) up to $10^{5}$.

Dyson (1968), on the other hand, analyses the situation by assuming that the globule is in hydrostatic equilibrium under self-gravitation, internal pressure, and a boundary 
pressure supplied by the HII streaming away from its surface in a D-critical I-front. No shocks are included, and a relation is derived between the mass and radius of the globule for a given external ionizing radiation field. This corresponds to the fact that the I-front (assumed to be D-critical) has a definite value of $p+\varrho v^{2}$ which must be balanced by the internal gas pressure. The theory of truncated isothermal spheres then leads to the mass-radius relation. As the globule evaporates, gravitational binding usually becomes less and less important; and confinement by external pressure, more important. Gravitational instability does not occur, and the whole globule is evaporated (on a time scale of 2 to $8 \times 10^{4} \mathrm{yr}$ per solar mass). On the other hand, globules rather close to instability initially can be set into collapse as time goes on.

The question of whether the front is D-critical was analyzed by Kahn (1969). He finds rather that the front is strong D-type, progressing subsonically into the globule and ejecting ionized gas supersonically into the HII region. He mentions the possibility that the detailed heating and cooling processes within the I-front may require a pressure wave to propagate ahead of the I-front, but otherwise omits deviations from hydrostatic equilibrium within the globule. Mendis $(1968,1969)$ analyzed the situation further, taking into account the fact that the globule is probably composed of $\mathrm{H}_{2}$ rather than $\mathrm{H}$ at the high densities contemplated $\left(10^{6} \mathrm{~cm}^{-3}\right)$. He concludes that the front is strong D-type for all stars earlier than $\mathrm{O}$.

Considerable effort has been expended on discovering whether elephant trunks (which may later become globules) can arise as a result of instability. The present consensus appears to be that instabilities (Rayleigh-Taylor; ionization front) are present but either yield parameters in disagreement with observation or are too weak (Mathews and O'Dell, 1969). Rather, it seems more natural to suppose that large density fluctuations are already present in the HI region before it is overrun by the I-front. Two possibilities suggest themselves. The first is that dense concentrations $\left(10^{4} \mathrm{~cm}^{-3}\right)$ are present in the cloud before the hot stars begin to shine. As their internal pressure must greatly exceed that of the normal intercloud medium, they must be gravitationally bound. In this case, it is hard to see why they do not collapse in the free-fall time $\left(10^{6} \mathrm{yr}\right)$, since isothermal spheres of this kind are unstable. Possibly they are actually collapsing, and represent unstable fragments of the original cloud which would have formed stars if the ultraviolet radiation field had not intervened.

Alternatively, one might identify them with the small cloudlets (1 to $30 M_{\odot}$ ) discovered by Heiles (1967a) in the interstellar medium. While the masses roughly agree, the densities do not. To account for this, we note that by the time the I-front makes the dense clouds visible, they have already been overrun by a shock front which will certainly compress them. The degree of compression can be estimated as $2 T_{2} / T_{1} \approx 20000 / T_{1}$, where $T_{2}$ is the Hil temperature and $T_{1}$ is the Hi temperature. Ordinarily $T_{1}$ is taken to be $100 \mathrm{~K}$, so the compression is a factor of 200 . In regions which are already denser than average, however, cooling processes are particularly effective, and $T_{1}$ might drop as low as $10 \mathrm{~K}$ as a result of cooling by $\mathrm{C}^{\circ}$. The resulting compression of a factor of 2000 , would result in a final density of $2 \times 10^{4} \mathrm{~cm}^{-3}$. 


\section{References}

Anand, K. C., Daniel, R. R., and Stephens, S. A.: 1968, Proc. Ind. Acad. Sci. 67, 267.

Bahcall, J. N. and Wolf, R. A.: 1968, Astrophys. J. 152, 701.

Burbidge, G. R., Gould, R. J., and Pottasch, S. R.: 1963, Astrophys. J. 138, 945.

Clark, B. G.: 1965, Astrophys. J. 142, 1398.

Davies, R. D. and Tovmassian, H. M.: 1963, Monthly Notices Roy. Astron. Soc. 127, 45.

Dibai, E. A.: 1963, Astron. Zh. 40, 606 (translation: 1964, Soviet Astron. 7, 606).

Dibai, E. A. and Kaplan, S. A.: 1964, Astron. Zh. 41, 652 (translation: 1965, Soviet Astron. 8, 520).

Dieter, N. H.: 1964, Astron. J. 69, 288.

Dieter, N. H.: 1967, Astrophys. J. 150, 435.

Dieter, N. H.: 1969, Publ. Astron. Soc. Pacific 81, 186.

Dyson, J. E.: 1968, Astrophys. Space Sci. 1, 388.

Field, G. B.: 1962, Interstellar Matter in Galaxies (ed. by L. Woltjer), Benjamin, New York, p. 183.

Field, G. B.: 1965, Astrophys. J. 142, 531.

Field, G. B.: 1969, Sixteenth Liège Symposium (June 1969), Institut d'Astrophysique, Liège.

Field, G. B. and Hutchins, J.: 1968, Astrophys. J. 153, 737.

Field, G. B. and Saslaw, W. C.: 1965, Astrophys. J. 142, 568.

Field, G. B. and Zachariades, C.: 1969, in preparation.

Field, G. B., Rather, J. D. G., Aannestad, P. A., and Orszag, S. A.: 1968, Astrophys. J. 151, 953.

Field, G. B., Goldsmith, D. W., and Habing, H. J.: 1969, Astrophys. J. Lett. 155, L149.

Flower, D. R.: 1969, Conference on Infra-Red and Microwave Radiation from Nebulae and Galaxies (July 1969), Institute of Theoretical Astronomy, Cambridge, England.

Gary, T., Holweger, H., Koch, M., and Richter, J.: 1969, Astron. Astrophys. 2, 446.

Girnstein, H. G. and Rohlfs, K.: 1964, Z. Astrophys. 59, 83.

Goldsmith, D. W.: 1969, The Formation and Equilibrium of Interstellar Clouds, Ph.D Dissertation, University of California, Berkeley, February, 1969 (also Astrophys. J., in press).

Goldsmith, D. W., Habing, H. J., and Field, G. B.: 1969, Astrophys. J. 158, 173.

Grahl, B. H., Hachenberg, O., and Mebold, U.: 1969, Beitr. Radioastron. 1, 3.

Habing, H. J.: 1969, Bull. Astron. Inst. Netherl. 20, 177.

Hayakawa, S., Nishimura, S., and Takayanagi, K.: 1961, Publ. Astron. Soc. Japan 13, 184.

Heiles, C.: 1967a, Astrophys. J. Suppl. 15, 97.

Heiles, C.: 1967b, Astron. J. 72, 1040.

Hjellming, R. M.: 1966, Astrophys. J. 143, 420.

Hjellming, R. M.: 1968, Interstellar Ionized Hydrogen (ed. by Y. Terzian), Benjamin, New York, p. 435 .

Hjellming, R. M., Gordon, C. P., and Gordon, K. J.: 1969, Astron. Astrophys. 2, 202.

Hoyle, F. and Harwit, M.: 1962, Publ. Astron. Soc. Pacific 74, 359.

Hoyle, F. and Ireland, J. G.: 1960, Monthly Notices Roy. Astron. Soc. 120, 173.

Hoyle, F., Narlikar, J. V., and Wheeler, T. A.: 1964, Nature 203, 914.

Hudson, P. D.: 1965, Monthly Notices Roy. Astron. Soc. 131, 23.

Hudson, P. D.: 1968, Monthly Notices Roy. Astron. Soc. 140, 255.

Kahn, F. D.: 1955, Gas Dynamics of Cosmic Clouds (ed. by J. M. Burgers and H. C. van de Hulst), North-Holland Publishing Co., Amsterdam, p. 60.

Kahn, F. D.: 1967, Radio Astronomy and the Galactic System (ed. by H. van Woerden), Academic Press, London, p. 95.

Kahn, F. D.: 1969, Physica 41, 172.

Kahn, F. D. and Dyson, J. E.: 1965, Ann. Rev. Astron. Astrophys. 3, 47.

Kahn, F. D. and Woltjer, L.: 1967, Radio Astronomy and the Galactic System (ed. by H. van Woerden), Academic Press, London, p. 117.

Kaler, J. B.: 1968, Interstellar Ionized Hydrogen (ed. by Y. Terzian), Benjamin, New York, p. 459. Kippenhahn, R. and Schlüter, A.: 1957, Z. Astrophys. 49, 73.

Kogure, T.: 1965, Publ. Astron. Soc. Japan 17, 385.

Kurt, V. G. and Sunyaev, R. A.: 1967, Astron. Zh. 44, 1157 (translation: 1968, Soviet Astron. 11, 928).

Lasker, B. M.: 1966a, Astrophys. J. 143, 700.

Lasker, B. M.: 1966b, Astrophys. J. 146, 571. 
Lasker, B. M.: 1967, Astrophys. J. 149, 23.

Layzer, D.: 1967, Fourteenth Liège Symposium (June 1966), Institut d'Astrophysique, Liège, p. 315.

Mathews, W. G.: 1965, Astrophys. J. 142, 1120.

Mathews, W. G.: 1966, Astrophys. J. 144, 206.

Mathews, W. G.: 1967, Astrophys. J. 147, 965.

Mathews, W. G. and O'Dell, C. R.: 1969, Ann. Rev. Astron. Astrophys. 7, 67.

Mendis, D. A.: 1968, Monthly Notices Roy. Astron. Soc. 141, 409.

Mendis, D. A.: 1969, Monthly Notices Roy. Astron. Soc. 142, 441.

Mezger, P. G.: 1968, Interstellar Ionized Hydrogen (ed. by Y. Terzian), Benjamin, New York, p. 477.

Minkowski, R.: 1968, Stars and Stellar Systems, Volume 7: Nebulae and Interstellar Matter (ed. by

B. M. Middlehurst and L. H. Aller), University of Chicago Press, Chicago, p. 623.

Oort, J. H.: 1954, Bull. Astron. Inst. Netherl. 12, 177.

Oort, J. H.: 1969, First Annual Nuffield Lecture (July 1969), Institute of Theoretical Astronomy, Cambridge, England.

Osterbrock, D. E.: 1965, Astrophys. J. 142, 1423.

Osterbrock, D. E.: 1969, Phil. Trans. Roy. Soc. London A264, 241.

Pagel, B.: 1969, Conference on Infra-Red and Microwave Radiation from Nebulae and Galaxies (July, 1969), Institute of Theoretical Astronomy, Cambridge, England.

Parker, E. N.: 1965, Astrophys. J. 142, 584.

Parker, E. N.: 1966, Astrophys. J. 145, 811.

Parker, E. N.: 1968a, Stars and Stellar Systems, Volume 7: Nebulae and Interstellar Matter (ed. by B. M. Middlehurst and L. H. Aller), University of Chicago Press, Chicago, p. 707.

Parker, E. N.: 1968b, in Proc. Intern. Conf. Cosmic Rays (November 1968), Tata Institute, Bombay.

Parker, E. N.: 1969, this volume, p. 168.

Peimbert, M.: 1967, Astrophys. J. 150, 825.

Penston, M. V., Munday, V. A., Stickland, D. J., and Penston, M. J.: 1969, Monthly Notices Roy. Astron. Soc. 142, 355.

Pikel'ner, S.: 1957, Astron. Zh. 34, 314 (translation: 1959, Soviet Astron. 1, 310).

Pikel'ner, S.: 1967, Astron. Zh. 44, 1915 (translation: 1968, Soviet Astron. 11, 737).

Pikel'ner, S.: 1968a, Ann. Rev. Astron. Astrophys. 6, 165.

Pikel'ner, S.: 1968b, Astrophys. Letters 2, 97.

Pottasch, S. R.: 1963, Astrophys. J. 437, 945.

Pottasch, S. R.: 1968, Bull. Astron. Inst. Netherl. 19, 469.

Radhakrishnan, V. and Murray, J. D.: 1969, Proc. Astron. Soc. Australia 1, 215.

Raimond, E.: 1966, Bull. Astron. Inst. Netherl. 18, 191.

Riegel, K.: 1967, Astrophys. J. 148, 87.

Rubin, R. H.: 1968, Astrophys. J. 153, 761.

Saraph, H. E., Seaton, M. J., and Shemming, J.: 1969, Phil. Trans. Roy. Soc. London A264, 77.

Shane, W. W. and Katgert, P.: 1967, Radio Astronomy and the Galactic System (ed. by H. van Woerden), Academic Press, London, p. 127.

Shcheglov, P. V.: 1968, Astrophys. Lett. 1, 145.

Shklovskii, I. S.: 1962, Astron. Zh. 39, 209 (translation: 1962, Soviet Astron. 6, 162).

Silk, J. I. and Werner, M. W.: 1969, Astrophys. J. 158, 185.

Spitzer, L., Jr.: 1951, Problems of Cosmical Aerodynamics, Central Air Documents Office, Dayton, Ohio, p. 31.

Spitzer, L., Jr.: 1956, Astrophys. J. 124, 20.

Spitzer, L., Jr.: 1968a, Diffuse Matter in Space, Interscience, New York.

Spitzer, L., Jr.: 1968b, Stars and Stellar Systems, Volume 7: Nebulae and Interstellar Matter (ed. by B. M. Middlehurst and L. H. Aller), University of Chicago Press, Chicago, p. 1.

Spitzer, L., Jr. and Savedoff, M. P.: 1950, Astrophys. J. 111, 593.

Spitzer, L., Jr. and Scott, E. H.: 1969, Astrophys. J. 157, 161.

Spitzer, L., Jr. and Tomasko, M. G.: 1968, Astrophys. J. 152, 971.

Stecher, T. P. and Williams, D. A.: 1967, Astrophys. J. Lett. 149, L29.

Swings, P.: 1965, Ann. Astrophys. 28, 703.

Terzian, Y., Mezger, P. G., and Schraml, J.: 1968, Astrophys. Lett. 1, 153.

Thompson, A. R., Colvin, R. S., and Hughes, M. P.: 1969, Astrophys. J. 158, 939.

Wade, C.: 1958, Rev. Mod. Phys. 30, 946. 
Wentzel, D. G.: 1967, Astrophys. J. 150, 453.

Zimmermann, H.: 1967, Fourteenth Liège Symposium (June 1966), Institut d'Astrophysique, Liège, p. 285.

Zimmermann, H.: 1968a, Astron. Nachr. 290, 193.

Zimmermann, H.: 1968b, Astron. Nachr. 290, 211. 